\title{
TYPICAL REPRESENTATIONS VIA FIXED POINT SETS IN BRUHAT-TITS BUILDINGS
}

\author{
PETER LATHAM AND MONICA NEVINS
}

\begin{abstract}
For a tame supercuspidal representation $\pi$ of a connected reductive $p$-adic group $G$, we establish two distinct and complementary sufficient conditions, formulated in terms of the geometry of the Bruhat-Tits building of $G$, for the irreducible components of its restriction to a maximal compact subgroup to occur in a representation of $G$ which is not inertially equivalent to $\pi$. The consequence is a set of broadly applicable tools for addressing the branching rules of $\pi$ and the unicity of $[G, \pi]_{G}$-types.
\end{abstract}

\section{INTRODUCTION}

One of the most fruitful tools for studying the (smooth, complex) representation theory of a reductive $p$-adic group $G=\mathbf{G}(F)$ is the theory of types. Given an irreducible representation $\pi$ of $G$ let $\mathfrak{s}_{\pi}$ denote its inertial equivalence class in the sense of [BK98]. An $\mathfrak{s}_{\pi}$-type $(J, \lambda)$ is an irreducible representation $\lambda$ of a compact open subgroup $J$ of $G$ such that containing $\lambda$ upon restriction to $J$ gives a necessary and sufficient condition for an irreducible representation of $G$ to be inertially equivalent to $\pi$. Constructions of types are central to many recent developments in the representation theory of $p$-adic groups, and in particular to the explicit constructions of supercuspidal representations [BK93, Mor99, Yu01, Ste08, SS08. Moreover, the theory of types has been shown to mirror much of the structure of the representation theory of $p$-adic groups in the more traditional sense: the Bushnell-Kutzko theory of covers gives an analogue of parabolic induction, and any instance of the local Langlands correspondence is expected to give rise to an inertial Langlands correspondence relating types to representations of the inertia group of $F$.

With types playing such a fundamental role in the representation theory of $p$-adic groups, it is natural to expect that they are somewhat hard to come by. Indeed the unicity of types is the expectation that, for a supercuspidal representation $\pi$ of $G$ admitting an $\mathfrak{s}_{\pi}$-type $(J, \lambda)$, all other $\mathfrak{s}_{\pi}$-types must arise from $(J, \lambda)$ by a series of minor representation-theoretic renormalizations (see Conjecture 6.1). The unicity of types is now known in many special cases, specifically for split general linear groups Pas05 and tame representations of special linear groups Lat16, Lat18, for depth-zero representations Lat17, and for most toral representations [LN19]; there are also some results towards the unicity of types for non-cuspidal representations Nad17, Nad19. Our goal in this paper is to study the unicity of types for "almost

Received by the editors May 13, 2021, and, in revised form, September 2, 2021.

2020 Mathematics Subject Classification. Primary 22E50.

Key words and phrases. Theory of types, $p$-adic groups, tame representations, Bruhat-Tits buildings, branching rules.

The first author's research was supported by the Heilbronn Institute for Mathematical Research. The second author's research was supported by a Discovery Grant from NSERC Canada. 
all" supercuspidal representations. Our result explicitly makes use of the geometry of the Bruhat-Tits building $\mathscr{B}(G)$ of $G$, and offers far more general results than have been obtained to date.

Specifically, we restrict attention to the supercuspidal representations and types constructed by $\mathrm{Yu}$ in [Yu01, which is applicable in the case that $\mathbf{G}$ is tamely ramified, and generalizes a previous construction due to Adler Ad198; following the terminology of [Fin21], we refer to such supercuspidal representations as tame. In almost all cases, restricting attention to these tame representations is a vacuous condition: Fintzen proved in [Fin21, extending earlier results of Kim [Kim07, that every supercuspidal representation of $G$ is tame if $p$ is coprime to the order of the Weyl group of $G$.

In order to describe the possible types contained within a tame supercuspidal representation $\pi$ of $G$, we consider more generally the question of branching rules for $\pi$ upon restriction to a maximal compact subgroup $K$ of $G$. An equivalent formulation of the unicity of types is the assertion that any $\mathfrak{s}_{\pi}$-type contained in $\left.\pi\right|_{K}$ (if there are any) must be induced from a type arising via Yu's construction. One of the impediments is that the description of the dual of $K$ remains an open problem, and complete branching rules, even for supercuspidal representations, have only been determined in a few special cases Cas73, Nev13.

We now give a brief description of our methods and results. Let $\pi$ be an irreducible, tame supercuspidal representation of $G$, constructed from a datum $\Sigma$ as in Yu01. Let $(J, \lambda)=\left(J(\Sigma), \lambda_{\Sigma}\right)$ denote the type associated to $\Sigma$ in Y Yu01, and fix a maximal compact subgroup of $G$, which must coincide with the stabilizer $G_{y}$ of some point $y \in \mathscr{B}(G)$.

Since $(J, \lambda)$ is an $\mathfrak{s}_{\pi}$-type, it follows from [BK98, Prop. 5.2] that each irreducible subquotient of the smooth representation $\Pi:=\mathrm{c}-\operatorname{Ind}_{J}^{G} \lambda$ is isomorphic to an unramified twist of $\pi$; in particular, $\left.\Pi\right|_{G_{y}}$ is isomorphic to a direct sum of copies of $\left.\pi\right|_{G_{y}}$. Therefore the components we wish to consider are those of the Mackey decomposition of $\left.\Pi\right|_{G_{y}}$ [Kut77, which can be written as

$$
\left.\Pi\right|_{G_{y}} \cong \bigoplus_{g \in G_{y} \backslash G / J} g\left(\operatorname{Ind}_{G_{g^{-1}} \cap{ }^{-1} \cap J}^{G_{g^{-1}}} \lambda\right)=\bigoplus_{g \in G_{y} \backslash G / J}{ }^{g} \tau(y, g),
$$

where each Mackey component $\tau(y, g)$ is a finite-dimensional representation of $G_{g^{-1} y}$ and $g^{-1} y$ ranges over the $G$-orbit of $y$ in $\mathscr{B}(G)$.

In this paper, we relate the capacity of $\tau(y, g)$ to contain an $\mathfrak{s}_{\pi}$-type to the position of the point $g^{-1} y$ in relation to the building-theoretic ingredients in the datum defining $\pi$ : the building of a twisted Levi subgroup $G^{0}$, and a vertex $x$ of $\mathscr{B}\left(G^{0}\right)$, viewed as a point in $\mathscr{B}(G)$. In particular, we introduce the following conjecture, which is a strengthening of the usual statement about the unicity of types (Conjecture 6.1).

Conjecture (Conjecture 6.5). Suppose that $\tau(y, g)$ contains a $\mathfrak{s}_{\pi}$-type. Then $g^{-1} y$ is a fixed point of the action of $J$ on $\mathscr{B}(G)$.

The sufficiency of this condition is shown in Proposition 6.4. As a step towards necessity, we prove the following two theorems, which are of independent interest. During this introduction, they are stated in a strictly weaker form for the sake of clarity.

Any point $z \in \mathscr{B}(G)$ has a unique closest point in $\mathscr{B}\left(G^{0}\right)$; this defines a projection map $\mathscr{B}(G) \rightarrow \mathscr{B}\left(G^{0}\right)$. Our first result describes a relationship between the 
structure of $\tau(y, g)$ (for fixed arbitrary $y$ and any $g \in G$ ) and the image of the point $g^{-1} y \in \mathscr{B}(G)$ under this projection map in relation to the point $x$ of the datum.

Theorem 1 (see Sections 7 and 8 ). Suppose that the projection of $g^{-1} y$ onto $\mathscr{B}\left(G^{0}\right)$ lies in a facet distinct from $x$. Then every irreducible component $\tau$ of $\tau(y, g)$ is contained in an irreducible, tame non-cuspidal representation of $G$, and hence is not an $\mathfrak{s}_{\pi}$-type.

More precisely, in Section 7 we prove this result under a weaker hypothesis about $J \cap G_{g^{-1} y}$. In Section 8, we relate this hypothesis to the projection map discussed above, and use this to both identify a large set of points in $\mathscr{B}(G)$ to which Theorem 1 applies, and a smaller subset to which it cannot.

For our second result, we identify a finite-index subgroup $H$ of $J$, equipped with a natural Moy-Prasad-like decreasing filtration by subgroups $H_{t}$, for $t \geq 0$. Each group $H_{t}$ fixes pointwise a compact neighbourhood $\mathscr{B}(G)^{H_{t}}$ of $x \in \mathscr{B}(G)$, and these neighbourhoods increase with $t$. Similarly, the filtration subgroups of the centre $Z^{0}$ of $G^{0}$ fix pointwise an increasing family of $G^{0}$-invariant neighbourhoods $\mathscr{B}(G)^{Z_{t}^{0}}$ of $\mathscr{B}\left(G^{0}\right)$.

Theorem 2 (see Section 9). Suppose that the geodesic from $x$ to $g^{-1} y$ meets a point of $\mathscr{B}(G)^{H_{t+}} \backslash \mathscr{B}(G)^{Z_{t}^{0}}$. Then every irreducible component $\tau$ of $\tau(y, g)$ must occur in the restriction of an irreducible representation which is not inertially equivalent to $\pi$. Hence $\tau$ is not an $\mathfrak{s}_{\pi}$-type.

We now describe the strategy of proof for each theorem. To prove Theorem 1 we construct, for each irreducible representation $\tau$ occuring in $\tau(y, g)$, a non-cuspidal representation containing $\tau$, by shifting from the point $x$ to a facet adjacent to $x$ determined by $\tau$. To this facet we are able to associate a type for a tame non-cuspidal representation following KY17, and show that this non-cuspidal representation must contain $\tau$. This argument is quite general, and of independent interest. It significantly generalizes the central argument of [Lat17, which applied only in the case that $\pi$ is depth-zero.

To prove Theorem 2] we perturb the simple character of our datum in a manner which does not alter the Mackey component under consideration (but also does not necessarily produce the simple character of a new representation). We then show that the existence of such a perturbation violates the capacity of the Mackey component to lie in the restriction of a single inertial class of supercuspidal representations. This generalizes an argument first presented in Pas05, and then carried out for all toral supercuspidal representations in LN19.

While these two results provide new, interesting, and explicit information regarding the branching rules of tame supercuspidal representations, our major motivation in proving these results is to develop building-theoretic tools towards a proof of the unicity of types which work in the most general setting currently available. Namely, with Theorems 1 and 2 in hand, proving Conjecture 6.5 now amounts to showing that for any point $g^{-1} y$ of $\mathscr{B}(G) \backslash \mathscr{B}(G)^{J}, g^{-1} y$ satisfies the hypotheses of at least one of the above two theorems. At the end of Section 9, we provide some context for why we believe this to be true, though (as we elaborate there) the proof reduces to some explicit and difficult open questions about the geometry of fixed point sets of tori acting on buildings.

Finally, let us contrast our approach with the work of Paškūnas in Pas05] for $G=\mathrm{GL}_{n}(F)$. In the language of our present paper, he partitioned $\mathscr{B}(G) \backslash \mathscr{B}(G)^{J}$ 
into two regions, A and B. When $g^{-1} y$ is in region A, the difference between $J$ and $J \cap G_{g^{-1} y}$ is detectable at depth zero whereas it is not if $g^{-1} y$ is in region B. For the latter, he applies an argument that we have generalized here to Theorem 9.2 in order to conclude that $\tau(y, g)$ cannot contain an $\mathfrak{s}_{\pi}$-type.

On the other hand, when $g^{-1} y$ is in region A, Paškūnas' approach is to show that the irreducible components of $\tau(y, g)$ occur in inertially inequivalent representations, and hence are not $[G, \pi]_{G}$-types. Moreover, in almost all cases Paškūnas in fact shows that the components $\tau(y, g)$ occur in inertially inequivalent supercuspidal representations; this argument relies on aspects of Deligne-Lusztig theory which are specific to $\mathbf{G L}_{n}\left(\mathbb{F}_{q}\right)$, and we do not offer a generalization here. In the sporadic cases where this approach cannot be made to work, Paškūnas shows that the components of $\tau(y, g)$ must intertwine with non-cuspidal representations, via a non-constructive argument.

The paper is organized as follows. We briefly set some notation in Section 2 before establishing the necessary background in Bruhat-Tits theory in Section 3 . In Section 4 we recap the general theory of types, before turning to the tame types which are the subject of this paper in Section 5. We lay out our strategy and define our Mackey components in Section 6. Section 7 is devoted to the statement and proof of Theorem 7.1. In Section 8 , we describe the projection onto a subbuilding and use these ideas to give two building-theoretic conditions under which Theorem 7.1 can or cannot hold. We also illustrate these with an example in $\mathbf{S p}_{4}(F)$, providing a lead-in to Section 9, which is devoted to the statement and proof of Theorem 9.2 , as well as a discussion of the geometry of its hypothesis.

\section{NotATiON}

We now establish some basic notation which will be used freely throughout the paper. Let $F$ be a field which is locally compact and complete relative to a normalized discrete valuation val. Let $\mathfrak{o} \subset F$ be its ring of integers, with maximal ideal $\mathfrak{p} \subset \mathfrak{o}$ and residue field $\mathfrak{f}=\mathfrak{o} / \mathfrak{p}$. We assume that the characteristic $p$ of $\mathfrak{f}$ is odd. Let $F^{\text {un }}$ denote a maximal unramified closure of $F$, contained within a separable algebraic closure $\bar{F} / F$.

Let $\mathbf{G}$ be a connected reductive algebraic group defined over $F$, and write $G=$ $\mathbf{G}(F)$ for its group of $F$-rational points, equipped with its locally profinite topology. We assume that the group $\mathbf{G}$ splits over a tamely ramified extension of $F$, and that $p$ is coprime to the order of the fundamental group of both $\mathbf{G}_{\bar{F}}^{\text {der }}$ and its reductive dual. We reserve the use of bold symbols for algebraic groups and group schemes defined over an o-algebra, latin characters for closed subgroups of $G$, and serif font for subgroups of the $\mathfrak{f}$-rational points of the special fibres of certain group schemes over $\mathfrak{o}$. We will often refer to a closed subgroup $H$ of $G$ of the form $\mathbf{H}(F)$ for some closed subgroup $\mathbf{H}$ of $\mathbf{G}$ as having a property if $\mathbf{H}$ has that property. In particular, this provides a notion of tori, parabolic subgroups and Levi subgroups of $G$ that we will frequently use.

On occasion, we will need to discuss disconnected algebraic groups, the connected component of the identity of which is reductive. In such cases, we extend all usual terminology in the obvious way: a torus of such a group is a subgroup which intersects with the connected component of the identity as a torus, and similarly for parabolic and Levi subgroups. 
For a locally profinite group $H$, we denote by $\operatorname{Rep}(H)$ the category of smooth complex representations of $H$, i.e. the category of (possibly infinite-dimensional) complex vector spaces $V$ equipped with an action of $H$ such that the stabilizer of any vector $v \in V$ is an open subgroup of $H$. Without exception, when we say "representation" during the remainder of the paper, we will mean "smooth complex representation".

We write $\operatorname{Ind}_{H}^{G}$ and $\mathrm{c}-\operatorname{Ind}_{H}^{G}$ for the induction and compact induction functors $\operatorname{Rep}(H) \rightarrow \operatorname{Rep}(G)$, respectively. While we will usually omit restriction functors from the notation, we will occasionally denote them by $\operatorname{Res}_{H}^{G}: \operatorname{Rep}(G) \rightarrow \operatorname{Rep}(H)$.

Given $H \subseteq G$ and $g \in G$, set ${ }^{g} H=\left\{g h g^{-1} \mid h \in H\right\}$ and for any $\rho \in \operatorname{Rep}(H)$, the corresponding representation ${ }^{g} \rho \in \operatorname{Rep}\left({ }^{g} H\right)$ is given on $k \in{ }^{g} H$ by ${ }^{g} \rho(k)=$ $\rho\left(g^{-1} \mathrm{~kg}\right)$.

\section{BRUhat-Tits ThEORY}

3.1. Structure theory and parahoric subgroups. First, note that for any algebraic torus $\mathbf{T}$ defined over $F$, there exists a finite-type Néron model of $\mathbf{T}$ ( $c f$. BLR90), which we denote by $\mathbf{T}_{\mathrm{b}}$. This is a smooth affine $\mathfrak{o}$-group scheme of finite type with generic fibre $\mathbf{T}_{\mathrm{b}} \times_{\text {Spec o }}$ Spec $F=\mathbf{T}$. We write $\mathbf{T}_{0}$ for the connected component of the identity. Then $T_{\mathrm{b}}=\mathbf{T}_{\mathrm{b}}(\mathfrak{o})$ is the maximal bounded subgroup of $T:=\mathbf{T}(F)$, and $T_{0}:=\mathbf{T}_{0}(\mathfrak{o})$ is a finite index subgroup of $T_{\mathrm{b}}$ called the parahoric subgroup of $T$. The Moy-Prasad filtration of $T$ is the decreasing filtration $\left\{T_{r} \mid r \geq 0\right\}$ of $T_{0}$ by open subgroups defined by

$$
T_{r}=\left\{t \in T_{0} \mid \operatorname{val}(\chi(t)-1) \geq r \text { for all } \chi \in X^{*}(\mathbf{T})\right\} ;
$$

here $X^{*}(\mathbf{T})$ denotes the character lattice of $\mathbf{T}$.

Now, and for the remainder of this section, suppose that $\mathbf{G}$ is a connected reductive group as in Section 2 with centre $Z(\mathbf{G})$. Choose a maximal $F$-split torus $\mathbf{S}$, contained in a maximal $F^{\mathrm{un}}$-split $F$-torus $\mathbf{S}^{\mathrm{un}}$. Since $\mathbf{G}$ is quasi-split over $F^{\mathrm{un}}$, the centralizer $\mathbf{T}$ of $\mathbf{S}^{\text {un }}$ is a maximal torus of $\mathbf{G}$. Write $\mathbf{C}$ for the centralizer of $\mathbf{S}$, which is a minimal Levi subgroup of $\mathbf{G}$; one has $\mathbf{C}=\mathbf{T}$ if and only if $\mathbf{G}$ is $F$-quasi-split.

Let $\Phi=\Phi(\mathbf{G}, \mathbf{S}, F)$ be the roots of $\mathbf{S}$ in $\mathbf{G}$ defined over $F$, and let $\Psi=\Psi(\mathbf{G}, \mathbf{S}, F)$ be an associated system of affine roots (relative to some base point, which we suppress). As described carefully in [Fin19, $\S 2.2, \S 2.3]$, the root subgroup $U_{\alpha} \subset G$, for $\alpha \in \Phi$, admits a filtration by compact open subgroups $U_{\psi}$, indexed by those $\psi \in \Psi$ with gradient $\alpha$.

Let $X_{*}(\mathbf{S})$ denote the group of cocharacters of $\mathbf{S}$. The affine space $\mathscr{A}=\mathscr{A}(\mathbf{G}, \mathbf{S}, F)$ $:=X_{*}(\mathbf{S}) \otimes_{\mathbb{Z}} \mathbb{R}$, called the apartment defined by $\mathbf{S}$, carries a hyperplane structure defined by $\Psi$. To each point $x \in \mathscr{A}$, Bruhat and Tits [BT84, §5.2] associated a parahoric group scheme, which is a smooth affine o-group scheme $\mathbf{G}_{x, 0}$ with the following properties (see, for example, YY15]):

(i) the generic fibre $\mathbf{G}_{x, 0} \times{ }_{\text {Spec o }}$ Spec $F$ of $\mathbf{G}_{x, 0}$ is equal to $\mathbf{G}$;

(ii) the special fibre $\mathbf{G}_{x, 0} \times{ }_{\text {Spec o }}$ Spec $\mathfrak{f}$ of $\mathbf{G}_{x, 0}$ is a connected reductive algebraic group defined over $\mathfrak{f}$ whose group of $\mathfrak{f}$-points we denote $\mathrm{G}_{x}$; and

(iii) the group $G_{x, 0}:=\mathbf{G}_{x, 0}(\mathfrak{o})$ of $\mathfrak{o}$-rational points of $\mathbf{G}_{x, 0}$ is compact and open in $G$, and is given by

$$
G_{x, 0}=\left\langle C_{0}, U_{\psi} \mid \psi \in \Psi: \psi(x) \geq 0\right\rangle,
$$


where $C_{0}$ is defined as above if $\mathbf{C}$ is a torus, and as in Remark 3.2, otherwise. Note that the group $C_{0}$ is independent of the choice of $x$ in either case.

3.2. The Bruhat-Tits building and point stabilizers. The (enlarged or extended) Bruhat-Tits building of $G$ is obtained by gluing together the apartments defined by all maximal $F$-split tori of $\mathbf{G}$. The action of $N_{G}(\mathbf{S}(F))$ on $\mathbf{S}(F)$ induces an action on the cocharacter lattice $X_{*}(\mathbf{S})$ [BT72, 6.2.10]. Set $\mathscr{B}(G)=$ $\mathscr{B}(\mathbf{G}, F):=(G \times \mathscr{A}(\mathbf{G}, \mathbf{S}, F)) / \sim$, where $(g, x) \sim\left(g^{\prime}, x^{\prime}\right)$ if and only if there exists an $n \in N_{G}(\mathbf{S}(F))$ such that $n x=x^{\prime}$ and $g^{-1} g^{\prime} n \in G_{x, 0}$ as in [BT72, 7.4(R)]. Then we identify $\{(g, x) \mid x \in \mathscr{A}\}$ with the apartment corresponding to ${ }^{g} \mathbf{S}$.

There is a canonical metric on $\mathscr{B}(G)$, known as the Bruhat-Tits metric, with respect to which $\mathscr{B}(G)$ is a non-positively curved (CAT $(0))$ geodesic metric space BT72, 3.1]. Given any two points $x, y \in \mathscr{B}(G)$, there exists an apartment $\mathscr{A}$ containing both. The geodesic $[x, y]$ is then a line segment in this apartment.

The left-regular action of $G$ on $G \times \mathscr{A}(\mathbf{G}, \mathbf{S}, F)$ descends to an action of $G$ on $\mathscr{B}(G)$ via isometries. Proposition 3.1 is originally due to Bruhat-Tits [BT84, 4.6.18] and proven directly in [Yu15, 9.3].

Proposition 3.1 (Bruhat-Tits). For each point $x \in \mathscr{B}(G)$, there exists a smooth affine o-group scheme $\mathbf{G}_{x}$ with finite component group such that:

(i) the group $G_{x}:=\mathbf{G}_{x}(\mathfrak{o})$ of $\mathfrak{o}$-rational points of $\mathbf{G}_{x}$ is compact and coincides with the stabilizer $\operatorname{Stab}_{G}(x)$ for $x \in \mathscr{B}(G)$; and

(ii) for any apartment $\mathscr{A}$ containing $x$, the connected component of the identity of $\mathbf{G}_{x}$ coincides with the parahoric group scheme $\mathbf{G}_{x, 0}$ defined relative to $\mathscr{A}$.

Remark 3.2. When $\mathbf{G}$ is not $F$-quasi-split and $\mathbf{C}$ is not a torus, choose a finite unramified extension $E / F$ such that $\mathbf{G}$ is $E$-quasi-split, so that the above construction applies. Then each apartment $\mathscr{A}(\mathbf{G}, \mathbf{S}, E)$ and thus the building $\mathscr{B}(\mathbf{G}, E)$ carry an action of $\operatorname{Gal}(E / F)$ such that we may identify $\mathscr{A}(\mathbf{G}, \mathbf{S}, E)^{\operatorname{Gal}(E / F)}=\mathscr{A}(\mathbf{G}, \mathbf{S}, F)$ and $\mathscr{B}(G)=\mathscr{B}(\mathbf{G}(E))^{\operatorname{Gal}(E / F)}$ [BT84, 5.1.25]. Further, the group $C_{0}$ (used to define $G_{x, 0}$ above) can be defined here by setting $C_{0}=\mathbf{C}(F) \cap \mathbf{G}(E)_{x, 0}$. This group is independent of the choice of point $x \in \mathscr{A}(\mathbf{G}, \mathbf{S}, E)$. Its filtration subgroups for $r \geq 0$ are similarly defined as $C_{r}=C_{0} \cap \mathbf{G}(E)_{x, r}$.

Remark 3.3. We will often need to take great care in distinguishing between the point stabilizer subgroups $G_{x}$ and the parahoric subgroups $G_{x, 0}$. One convenient method of describing the difference between these two groups is to use the Kottwitz homomorphism, as defined in Kot97. This is a homomorphism $\kappa$ from $G$ to the algebraic fundamental group $\pi_{1}^{\text {alg }}(G)$ of $G$, as in Bor98, which has the property that, for any $x \in \mathscr{B}(G)$, one has $G_{x} \cap \operatorname{ker} \kappa=G_{x, 0}$ [PR08, Appendix]. In fact, the Kottwitz homomorphism can be used to describe $G_{x} \backslash G_{x, 0}$ as the set of $g \in G_{x}$ for which $\kappa(g)$ is a non-trivial torsion element of $\pi_{1}^{\mathrm{alg}}(G)$.

3.3. Moy-Prasad filtrations and Levi subgroups. For any $x \in \mathscr{B}(G)$, choose an apartment $\mathscr{A}=\mathscr{A}(\mathbf{G}, \mathbf{S}, F)$ containing $x$. Moy and Prasad defined a filtration $\left\{G_{x, r} \mid r \geq 0\right\}$ of $G_{x, 0}$ by open normal subgroups by setting

$$
G_{x, r}=\left\langle C_{r}, U_{\psi} \mid \psi \in \Psi(\mathbf{G}, \mathbf{S}, F): \psi(x) \geq r\right\rangle .
$$

A convenient observation is that the Moy-Prasad filtration subgroups $G_{x, r}$ are actually schematic, which is to say that there are (uniquely determined) smooth 
affine o-group schemes $\mathbf{G}_{x, r}$ with generic fibre $\mathbf{G}$ such that $\mathbf{G}_{x, r}(\mathfrak{o})=G_{x, r}$ Yu15, 8.6]. In particular, for each $\operatorname{root} \alpha \in \Phi(\mathbf{G}, \mathbf{S}, F)$, each $x \in \mathscr{A}$ and each $r \in \mathbb{R}$ there is a smooth affine $\mathfrak{o}$-group scheme $\mathbf{U}_{\alpha, x, r}$ whose group of $\mathfrak{o}$-rational points is $\bigcup_{\psi} U_{\psi}$, where this union is over all affine roots $\psi$ of $\mathscr{A}$ with gradient $\alpha$ that satisfy the condition $\psi(x) \geq r$ Y Yu15, 6.2].

Write $G_{x, r+}=\bigcup_{s>r} G_{x, s}$ and $G_{x, r: r+}=G_{x, r} / G_{x, r+}$. Then $G_{x, 0: 0+}=\mathrm{G}_{x}$ is a finite group of Lie type, as in Section 3.1(ii), whereas $G_{x, r: r+}$ is an abelian $p$-group for $r>0$.

The root system $\Phi_{x}$ of $\mathrm{G}_{x}$ is defined by (gradients of) the affine roots that vanish at $x$ Fin19, $\S 2.4, \S 2.5]$. If $y$ lies in a facet of $\mathscr{B}(G)$ that contains $x$ in its closure, then $G_{x, 0+} \subset G_{y, 0} \subset G_{x, 0}$ and $\Phi_{y} \subset \Phi_{x}$; in fact the quotient $G_{y, 0} / G_{x, 0+}$ identifies with a parabolic subgroup $\mathrm{P}$ of $\mathrm{G}_{x}$. Further, by [MP96, §6.3, Proposition 6.4], we may associate to $y$ an $F$-Levi subgroup $\mathbf{M}$ of $\mathbf{G}$ such that $\mathrm{M}_{y} \cong \mathrm{G}_{y}$ is isomorphic to an $\mathfrak{f}$-Levi factor of $\mathrm{P}$. Conversely, note that for each proper parabolic subgroup $\mathrm{P}$ of $\mathrm{G}_{x}$ there is a $\mathrm{g} \in \mathrm{G}_{x}$ such that ${ }^{\mathrm{g}} \mathrm{P}$ is in the above standard form. If we choose a lift $g \in G_{x, 0}$ of $\mathrm{g}$, then $g^{-1} \mathscr{A}$ contains $x$ and, by the above, a point $y$ in a facet whose closure contains $x$ such that $\mathrm{P} \cong G_{y, 0} / G_{x, 0+}$.

3.4. The reduced building and other properties. The reduced building of $G$ is $\mathscr{B}^{\text {red }}(G)=\mathscr{B}\left(G^{\text {der }}\right)$, where $G^{\text {der }}=\mathbf{G}^{\text {der }}(F)$, for $\mathbf{G}^{\text {der }} \subset \mathbf{G}$ the derived subgroup. One has $\mathscr{B}(G)=\mathscr{B}^{\text {red }}(G) \times X_{*}(Z(\mathbf{G})) \otimes_{\mathbb{Z}} \mathbb{R}$, and so there is a projection map $\mathscr{B}(G) \rightarrow \mathscr{B}^{\text {red }}(G)$, which we denote by $x \mapsto[x]$. Write $\widetilde{[x]}$ for the fibre of this projection over $[x]$.

For any $x, y \in \mathscr{B}(G)$ such that $[x]=[y]$, we have that $G_{x}=G_{y}$. The induced action of $G$ on $\mathscr{B}^{\text {red }}(G)$ is such that the stabilizer $G_{[x]}$ of $[x] \in \mathscr{B}^{\text {red }}(G)$ is only a compact-modulo-centre subgroup of $G$. Its maximal compact subgroup is $G_{x}$ and in fact $G_{[x]}=N_{G}\left(G_{x, 0}\right)$.

An advantage of working with $\mathscr{B}^{\text {red }}(G)$ is that it possesses vertices; we often refer to a point in $\mathscr{B}(G)$ as a vertex if its image $[x]$ is one. For example, if $x$ is a vertex then $G_{x, 0}$ is a maximal parahoric subgroup.

By Proposition 3.1 $G_{x}$ is the stabilizer of $x \in \mathscr{B}(G)$ under the action of $G$. More generally, for a subset $\Omega \subset \mathscr{B}(G)$, we use the notation

$$
G_{\Omega}=\{g \in G \mid g x=x, \forall x \in \Omega\}
$$

for the pointwise stabilizer of $\Omega$. Given a subgroup $H$ of $G$, we write $\mathscr{B}(G)^{H}$ for the set of fixed points, which we may equivalently write as as $\left\{x \in \mathscr{B}(G) \mid H \subset G_{x}\right\}$.

Since $\mathscr{B}(G)$ is a $\operatorname{CAT}(0)$ space, if $g \in G$ fixes both $x$ and $y$, then it fixes the geodesic $[x, y]$. This has several key consequences. For one, if $z \in[x, y]$ then $G_{x} \cap G_{y} \subset G_{z}$, a fact which we will use frequently in the sequel. For another, it follows that for any $H, \mathscr{B}(G)^{H}$ is convex; its image in $\mathscr{B}^{\text {red }}(G)$ is bounded if, for example, $H$ is compact open.

\section{THE THEORY OF TYPES}

In this section, we recall the theory of types in the abstract sense laid out in BK98; the objective of this paper is to describe to what extent all such types, in the tame case, may be described in terms of the types constructed in [Yu01. 
4.1. Bernstein decomposition. A cuspidal pair in $G$ is a pair $(M, \rho)$ consisting of a Levi subgroup $M$ of $G$ and an irreducible supercuspidal representation $\rho$ of $M$. Given $\pi$ a smooth irreducible representation of $G$, Jacquet's theorem implies there exists a unique $G$-conjugacy class of cuspidal pairs $(M, \rho)$ such that $\pi$ is isomorphic to a subquotient of $\operatorname{Ind}_{P}^{G} \rho$ for some parabolic subgroup $P$ of $G$ with Levi factor $M$. We call this conjugacy class the cuspidal support of $\pi$.

We say that cuspidal pairs $(M, \rho)$ and $\left(M^{\prime}, \rho^{\prime}\right)$ are $G$-inertially equivalent if there exists an unramified character $\omega$ of $M^{\prime}$ and a $g \in G$ such that ${ }^{g} M=M^{\prime}$ and ${ }^{g} \rho \simeq \rho^{\prime} \otimes \omega$. We write $\mathfrak{s}=[M, \rho]_{G}$ for the $G$-inertial equivalence class of $(M, \rho)$. The inertial support of an irreducible representation of $G$ is the inertial equivalence class of its cuspidal support. We say that two irreducible representations are inertially equivalent if they have the same inertial support, and write $\mathfrak{I}(\pi)$ for the inertial equivalence class of $\pi$.

Write $\mathfrak{B}(G)$ for the set of $G$-inertial equivalence classes of cuspidal pairs. Given any subset $\mathfrak{S}$ of $\mathfrak{B}(G)$, denote by $\operatorname{Rep}^{\mathfrak{S}}(G)$ the full subcategory of $\operatorname{Rep}(G)$ consisting of those representations every irreducible subquotient of which has inertial support contained in $\mathfrak{S}$. By a theorem of Bernstein [Ber84], we have a categorical decomposition

$$
\operatorname{Rep}(G)=\prod_{\mathfrak{s} \in \mathfrak{B}(G)} \operatorname{Rep}^{\mathfrak{s}}(G)
$$

over the singleton subsets of $\mathfrak{B}(G)$.

\subsection{Types and covers.}

Definition 4.1. Let $\mathfrak{S} \subset \mathfrak{B}(G)$ be a finite set, and let $(J, \lambda)$ be a pair consisting of an irreducible representation $\lambda$ of a compact open subgroup $J$ of $G$.

(i) We say that $(J, \lambda)$ is $\mathfrak{S}$-typical if, for any irreducible representation $\pi$ of $G$ such that $\operatorname{Hom}_{J}(\lambda, \pi) \neq 0$, one must have $\pi \in \operatorname{Rep}^{\mathfrak{S}}(G)$.

(ii) We say that $(J, \lambda)$ is an $\mathfrak{S}$-type if it is $\mathfrak{S}$-typical and every irreducible representation $\pi$ in $\operatorname{Rep}^{\mathfrak{S}}(G)$ satisfies $\operatorname{Hom}_{J}(\lambda, \pi) \neq 0$.

As simple applications of Frobenius reciprocity and the transitivity of compact induction, one immediately deduces a few properties of types:

(i) For any $g \in G$, the pair $\left({ }^{g} J,{ }^{g} \lambda\right)$ is an $\mathfrak{S}$-type if and only if $(J, \lambda)$ is an S-type.

(ii) If $(J, \lambda)$ is an S-type, $K \supset J$ is a compact open subgroup of $G$ and $\tau$ is an irreducible representation of $K$ satisfying $\operatorname{Hom}_{J}(\lambda, \tau) \neq 0$, then $(K, \tau)$ is S-typical.

(iii) If $\mathfrak{s}=[G, \pi]_{G}$ is an inertial equivalence class of supercuspidal representations of $G$, then $(J, \lambda)$ is an $\mathfrak{s}$-type if and only if it is $\mathfrak{s}$-typical.

In particular, combining (ii) and (iii) shows that any irreducible representation of

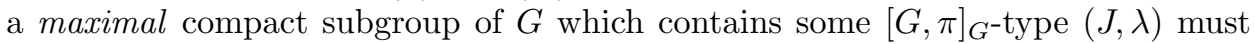
itself be a $[G, \pi]_{G}$-type.

Suppose now that $M$ is a Levi subgroup of $G$, and $\left(J_{M}, \lambda_{M}\right)$ an $\mathfrak{S}_{M}$-type, where $\mathfrak{S}_{M}=\left\{\left[L_{i}, \rho_{i}\right]_{M}\right\} \subset \mathfrak{B}(M)$ is a finite set. In BK98, Bushnell and Kutzko define the notion of a cover $(J, \lambda)$ of $\left(J_{M}, \lambda_{M}\right)$ which, if it exists, is an $\mathfrak{S}$-type, where $\mathfrak{S}=\left\{\left[L_{i}, \rho_{i}\right]_{G}\right\}$. In this case, we have the following result.

Lemma 4.2. Suppose that $\left(J_{M}, \lambda_{M}\right)$ is an $[M, \rho]_{M}$-type and $(J, \lambda)$ is a $G$-cover of $\left(J_{M}, \lambda_{M}\right)$. Then $\mathrm{c}-\operatorname{Ind}_{J}^{G} \lambda$ has a filtration by subrepresentations such that each 
successive quotient is a finite-length representation isomorphic to c-Ind ${ }_{P}^{G} \rho^{\prime}$ for some $\rho^{\prime} \in \mathfrak{I}(\rho)$ and some parabolic subgroup $P$ of $G$ with Levi factor $M$.

Proof. Fix a parabolic subgroup $P=M N$ of $G$ with Levi factor $M$. By Blo05,

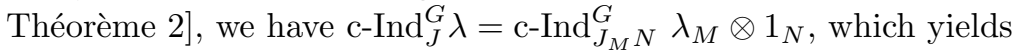

$$
\mathrm{c}-\operatorname{Ind}_{J}^{G} \lambda=\operatorname{Ind}_{M N}^{G}\left(\mathrm{c}-\operatorname{Ind}_{J_{M}}^{M} \lambda_{M}\right) \otimes 1_{N} .
$$

The claim then follows from [BK98, Proposition 5.2].

\section{KIM-YU TYPES}

In this section, we recall the construction of types for tame representations by $\mathrm{Yu}$ and Kim as given in YYu01 and [KY17]. We largely follow the treatment given in [HM08, but we depart slightly from their notational conventions in some cases, so as to use $J$ for the compact open subgroups supporting types, as in [BK98. For the reader familiar with these constructions the main substitutions are (a) $J$ in place of $K$ as the root letter in notation to denote an open compact-mod-centre subgroup, (b) $\mathcal{J}$ in place of $J$ as the root letter for the groups like $\left(G^{i}, G^{i+1}\right)_{r_{i}, s_{i}}$, (c) $H_{+}$in place of $K_{+}$as the largest subgroup on which the type acts via a character.

\subsection{Data.}

Definition 5.1. A twisted Levi sequence in $\mathbf{G}$ is a sequence $\overrightarrow{\mathbf{G}}=\left(\mathbf{G}^{0}, \ldots, \mathbf{G}^{d}\right)$ of closed $F$-subgroups $\mathbf{G}^{i}$ of $\mathbf{G}$ such that $\mathbf{G}^{0} \subsetneq \mathbf{G}^{1} \subsetneq \cdots \subsetneq \mathbf{G}^{d}=\mathbf{G}$ and such that there exists a finite algebraic extension $E / F$ such that each group $\mathbf{G}^{i}(E)$ is a Levi subgroup of $\mathbf{G}(E)$. We say that $\overrightarrow{\mathbf{G}}$ splits over $E$. A generalized twisted Levi sequence is defined similarly but without the condition that adjacent elements be distinct.

We require the definition of a (not necessarily supercuspidal) datum given in [KY17, 7.2]. Due to their complexity, (iii), (vii) and (viii) are not fully defined below. Instead, the generic embeddings of viiil will be discussed in detail where they are used in the proof of Lemma 7.2, the genericity of the characters in (vii) does not come into question in our construction, so we do not review it; but we will recap the construction of the groups $\mathbf{M}^{i}$ of (iii) in Remark 5.3

Definition 5.2. A datum in $G$ is a tuple $\Sigma=\left(\left(\overrightarrow{\mathbf{G}}, \mathbf{M}^{0}\right),(x,\{\iota\}),\left(J_{M}^{0}, \sigma\right), \vec{r}, \vec{\phi}\right)$ consisting of

(i) a twisted Levi sequence $\overrightarrow{\mathbf{G}}$ in $\mathbf{G}$ which splits over a tamely ramified extension $E / F$;

(ii) a Levi subgroup $\mathbf{M}^{0}$ of $\mathbf{G}^{0}$, which defines Levi subgroups $\mathbf{M}^{i}$ of $\mathbf{G}^{i}$ such that $\overrightarrow{\mathbf{M}}$ is a generalized twisted Levi sequence of $\mathbf{M}=\mathbf{M}^{d}$ as in [KY17, §2.4];

(iii) a point $x \in \mathscr{B}\left(M^{0}\right)$ for which $M_{x, 0}^{0}$ is a maximal parahoric subgroup of $M^{0}=\mathbf{M}^{0}(F)$

(iv) a compact open subgroup $J_{M}^{0}$ of $M_{x}^{0}$ containing $M_{x, 0}^{0}$;

(v) an irreducible representation $\sigma$ of $J_{M}^{0}$ such that $\left.\sigma\right|_{M_{x, 0}^{0}}$ is a sum of cuspidal representations of $M_{x, 0: 0+}^{0}$;

(vi) a sequence $\vec{r}=\left(r_{0}, \ldots, r_{d}\right)$ of positive real numbers satisfying $r_{0}<r_{1}<$ $r_{2}<\cdots<r_{d-1} \leq r_{d}$ if $d>0$ and $0 \leq r_{0}$ if $d=0$ 
(vii) a sequence $\vec{\phi}=\left(\phi^{0}, \ldots, \phi^{d}\right)$ of (quasi-)characters $\phi^{i}$ of $G^{i}$, such that $\phi^{i}$ is $G^{i+1}$-generic of depth $r_{i}$ relative to $x$ (and thus relative to any $y \in \mathscr{B}\left(G^{i}\right)$ ), for $0 \leq i \leq d-1$, in the sense of [HM08, Definition 3.9]; in the case that $r_{d}=r_{d-1}$, we also require that the character $\phi^{d}$ is trivial; and

(viii) an $\vec{s}$-generic (relative to $x$ ) diagram of embeddings $\{\iota\}$ of the buildings $\mathscr{B}\left(M^{i}\right)$ and $\mathscr{B}\left(G^{i}\right)$ (following the given inclusions of groups) in the sense of [KY17, 3.5], where $\vec{s}=\left(s_{-1}, s_{0}, s_{1}, \ldots, s_{d-1}\right)$ with $s_{-1}=0$ and $s_{i}=r_{i} / 2$ for $0 \leq i<d$.

In the case that $M^{0}=G^{0}$ and $Z\left(\mathbf{G}^{0}\right) / Z(\mathbf{G})$ is anisotropic, we say that $\Sigma$ is a supercuspidal datum; this is the case considered in Yu01. For a supercuspidal datum, the choices of embeddings $\{\iota\}$ are unimportant [Yu01, Remark 3.4], so we instead write briefly $\Sigma=\left(\overrightarrow{\mathbf{G}}, x, J^{0}, \sigma, \vec{r}, \vec{\phi}\right)$, omitting the group $J^{0}$ of (iv) when $J^{0}=G_{x}^{0}$ is maximal.

Remark 5.3. The groups $\mathbf{M}^{i}$ appearing in (iii) above are defined in [KY17, §2.4] as follows. Let $Z_{\mathbf{s}}\left(\mathbf{M}^{0}\right)^{\circ}$ be the maximal $F$-split torus in the centre of $\mathbf{M}^{0}$. For each $0 \leq i \leq d$, let $\mathbf{M}^{i}$ be the $\mathbf{G}^{i}$-centralizer of $Z_{\mathbf{S}}\left(\mathbf{M}^{0}\right)^{\circ}$. This defines a sequence $\overrightarrow{\mathbf{M}}=\left(\mathbf{M}^{0}, \mathbf{M}^{1}, \ldots, \mathbf{M}^{d}\right)$ that, as shown in [KY17, $\left.\S 2.4\right]$, is a generalized twisted Levi sequence in $\mathbf{M}=\mathbf{M}^{d}$, defined over $F$ and split over $E$. Moreover, the quotient of the centers $Z\left(\mathbf{M}^{0}\right) / Z\left(\mathbf{M}^{d}\right)$ is anisotropic over $F$. We set $M^{i}=\mathbf{M}^{i}(F)$.

5.2. Groups associated to the datum. For the remainder of the section, fix a datum $\Sigma=\left(\left(\overrightarrow{\mathbf{G}}, \mathbf{M}^{0}\right),(x,\{\iota\}),\left(J_{M}^{0}, \sigma\right), \vec{r}, \vec{\phi}\right)$. Set $J_{G}^{0}=J_{M}^{0} G_{x, 0}^{0}$; this is a subgroup of $G_{x}^{0}$ such that $J_{G}^{0} / G_{x, 0+}^{0}=J_{M}^{0} / M_{x, 0+}^{0}$.

For each $0 \leq i \leq d$, define compact open subgroups of $G^{i}$ by

$$
\begin{aligned}
J^{i}(\Sigma, G) & =J_{G}^{0} G_{x, s_{0}}^{1} \cdots G_{x, s_{i-1}}^{i} ; \text { and } \\
H^{i}(\Sigma, G) & =J_{G}^{0} G_{x, s_{0}+}^{1} \cdots G_{x, s_{i-1}+}^{i} .
\end{aligned}
$$

These are in fact the $\mathfrak{o}$-points of the analogous affine normal $\mathfrak{o}$-group schemes, each of whose generic fibres are equal to $\mathbf{G}^{i}$. Where the pair $(\Sigma, G)$ is understood, we may choose to omit it from the notation.

The induced Moy-Prasad filtration on each of these subgroups is defined, for each $t \geq 0$ and any $0 \leq i \leq d$, as

$$
J_{t}^{i}:=J^{i} \cap G_{x, t}, \text { and } H_{t}^{i}:=H^{i} \cap G_{x, t} .
$$

We abbreviate $J_{+}^{i}:=J_{0+}^{i}$ and $H_{+}^{i}=H_{0+}^{i}$. Then we have a tower of compact open subgroups of $G^{i}$ given by

$$
H_{+}^{i} \subseteq J_{+}^{i} \subseteq J_{0}^{i} \subseteq J^{i}
$$

such that $J_{0}^{i} / J_{+}^{i} \cong G_{x, 0: 0+}^{0}$ for every $0 \leq i \leq d$. By [KY17, Proposition 4.3(b)], we also have

$$
J^{i} / J_{+}^{i} \cong J_{M}^{0} / M_{x, 0+}^{0} \cdot
$$

For each $0 \leq i<d-1$, our next groups are defined first as subgroups of $\mathbf{G}^{i+1}(E)$. Let $\mathbf{T}$ be a maximal $E$-split $F$-torus of $\mathbf{G}^{0}$ and for each $0 \leq j \leq d$ set $\Phi^{j}=\Phi\left(\mathbf{G}^{j}, \mathbf{T}, E\right)$. Then we may define

$$
\begin{aligned}
& \mathcal{g}^{i+1}\left(\Sigma, G, \mathfrak{o}_{E}\right):=\left\langle\mathbf{T}_{r_{i}}\left(\mathfrak{o}_{E}\right), \mathbf{U}_{\alpha, x, r_{i}}\left(\mathfrak{o}_{E}\right), \mathbf{U}_{\beta, x, s_{i}}\left(\mathfrak{o}_{E}\right) \mid \alpha \in \Phi^{i}, \beta \in \Phi^{i+1} \backslash \Phi^{i}\right\rangle ; \text { and } \\
& \mathcal{j}_{+}^{i+1}\left(\Sigma, G, \mathfrak{o}_{E}\right):=\left\langle\mathbf{T}_{r_{i}}\left(\mathfrak{o}_{E}\right), \mathbf{U}_{\alpha, x, r_{i}}\left(\mathfrak{o}_{E}\right), \mathbf{U}_{\beta, x, s_{i}+}\left(\mathfrak{o}_{E}\right) \mid \alpha \in \Phi^{i}, \beta \in \Phi^{i+1} \backslash \Phi^{i}\right\rangle .
\end{aligned}
$$


These define two more compact open subgroups of $G^{i+1}$

$$
\mathcal{J}^{i+1}(\Sigma, G):=\mathcal{J}^{i+1}\left(\Sigma, G, \mathfrak{o}_{E}\right) \cap G, \text { and } \mathcal{J}_{+}^{i+1}(\Sigma, G):=\mathcal{J}_{+}^{i+1}\left(\Sigma, G, \mathfrak{o}_{E}\right) \cap G .
$$

In the literature, the preceding is often abbreviated by writing

$$
g^{i+1}(\Sigma, G)=\left(G^{i}, G^{i+1}\right)_{r_{i}, s_{i}} \text { and } \partial_{+}^{i+1}(\Sigma, G)=\left(G^{i}, G^{i+1}\right)_{r_{i}, s_{i}+},
$$

which we will use in the sequel for convenience. Again, we may omit $(\Sigma, G)$ where this is clear from context.

Note that $J^{i+1}$ can be thought of as a kind of complement to $J^{i}$ in $J^{i+1}$ in the sense that

$$
J^{i} g^{i+1}=J^{i+1}
$$

but crucially, $J^{i} \cap \mathcal{J}^{i+1}=G_{x, r_{i}}^{i}$ is non-trivial. These groups give us an alternate description of $H$ [HM08, $\S 3]$ as

$$
H=J_{G}^{0} \partial_{+}^{1} \ldots \partial_{+}^{d} .
$$

Finally, from the datum $\Sigma$ (and Remark 5.3) we can also extract the tuple $\Sigma_{M}:=\left(\overrightarrow{\mathbf{M}}, x, J_{M}^{0}, \sigma, \vec{r}, \vec{\phi}\right)$. Lemma 5.4 summarizes results proven in [KY17, 7.4], using $\mathrm{Yu} 01$.

Lemma 5.4. The tuple $\Sigma_{M}$ is a supercuspidal datum in $M$ and, for $0 \leq i \leq d$, one has equalities

$$
\begin{aligned}
J^{i}(\Sigma, M) & :=J^{i}(\Sigma, G) \cap M^{i}=J^{i}\left(\Sigma_{M}, M\right) \text { and } \\
H^{i}(\Sigma, M) & :=H^{i}(\Sigma, G) \cap M^{i}=H^{i}\left(\Sigma_{M}, M\right) .
\end{aligned}
$$

Notation. Of the various groups associated to a datum, we will most frequently work with those with index $i=d$; when working with such groups, we often omit this index from the notation, writing $J(\Sigma, G)=J^{i}(\Sigma, G)$, etc.

5.3. The types $\left(J(\Sigma, G), \lambda_{\Sigma}\right)$ and $\left(J(\Sigma, M), \lambda_{\Sigma}\right)$. Continuing with the notation as above, the restriction of the character $\phi^{i}$ to $G_{x, r_{i}}^{i}$ admits an extension $\hat{\phi}^{i}$ to $\mathrm{J}_{+}^{i+1}(\Sigma, G)$. We write $\mathcal{N}^{i+1}(\Sigma, G)=\operatorname{ker} \hat{\phi}^{i}$ Yu01, §4]. Set

$$
\begin{aligned}
\mathcal{H}^{i+1}(\Sigma, G) & :=\mathcal{J}^{i+1}(\Sigma, G) / \mathcal{N}^{i+1}(\Sigma, G), \\
\mathcal{W}^{i+1}(\Sigma, G) & :=\mathcal{J}^{i+1}(\Sigma, G) / \mathcal{J}_{+}^{i+1}(\Sigma, G), \quad \text { and } \\
\mathcal{Z}^{i+1}(\Sigma, G) & :=\mathcal{J}_{+}^{i+1}(\Sigma, G) / \mathcal{N}^{i+1}(\Sigma, G) .
\end{aligned}
$$

Let us omit $(\Sigma, G)$ for brevity. Lemma 5.5 summarizes results proven in [Yu01, §11].

Lemma 5.5. The quotient $\mathcal{W}^{i+1}$ is a finite-dimensional $\mathbb{F}_{p}$-vector space. Given $j, j^{\prime} \in \mathrm{j}^{i+1}$, the commutator $\left[j, j^{\prime}\right]$ is contained in $\mathrm{j}_{+}^{i+1}$, and $\hat{\phi}^{i} \circ[-,-]$ defines a nondegenerate symplectic form on $\mathcal{W}^{i+1}$. Moreover, the quotient $\mathcal{H}^{i+1}$ is a Heisenberg p-group with centre $Z^{i+1}$.

As elaborated carefully in [HM08, 3.25-26], we may define the Heisenberg-Weil lift $\hat{\eta}$ of $\hat{\phi}^{i}$ to the group $\operatorname{Sp}\left(\mathcal{W}^{i+1}\right) \ltimes \mathcal{H}^{i+1}$. Since $J^{i}=J^{i}(\Sigma, G)$ acts on $g^{i+1}$ by conjugation, preserving the symplectic form, we have a map $J^{i} \ltimes J^{i+1} \rightarrow \operatorname{Sp}\left(\mathcal{W}^{i+1}\right) \ltimes$ $\mathcal{H}^{i+1}$. With respect to this map we set, for each $g j \in J^{i} g^{i+1}$,

$$
\kappa^{i}(g j)=\phi^{i}(g) \hat{\eta}(g, j) .
$$

This is well-defined, and by (5.3) gives an irreducible representation of $J^{i+1}$. 
Set $\kappa^{d}=\phi^{d}$. Inflate each of the remaining representations $\kappa^{i}$ to a representation of $J(\Sigma, G):=J^{d}(\Sigma, G)$. Using (5.1), we may also inflate $\sigma$ to a representation of $J(\Sigma, G)$. Set $\kappa_{\Sigma}=\bigotimes_{i=0}^{d} \kappa^{i}$ and define

$$
\lambda_{\Sigma}=\sigma \otimes \kappa_{\Sigma}
$$

Proposition 5.6 ([KY17, Theorem 7.5]). The representation $\lambda_{\Sigma}$ restricts irreducibly to $J(\Sigma, M)=J(\Sigma, G) \cap M$. The pair $\left(J(\Sigma, M), \lambda_{\Sigma}\right)$ is an $\mathfrak{S}$-type for some finite set of inertial equivalence classes of $M$ supported on $M$. Moreover, the pair $\left(J(\Sigma, G), \lambda_{\Sigma}\right)$ is a $G$-cover of $\left(J(\Sigma, M), \lambda_{\Sigma}\right)$. If $J_{M}^{0}=M_{x}^{0}$, then $\mathfrak{S}=\left\{\left[M, \pi_{\Sigma, M}\right]_{M}\right\}$ for some irreducible supercuspidal representation $\pi_{\Sigma, M} \hookrightarrow \mathrm{c}-\operatorname{Ind}_{J(\Sigma, M)}^{M} \lambda_{\Sigma}$, and $\left(J(\Sigma, G), \lambda_{\Sigma}\right)$ is an $\left[M, \pi_{\Sigma, M}\right]_{G}$-type.

In the special case that $\Sigma$ is a supercuspidal datum and $J_{G}^{0}=G_{x}^{0}$ is maximal, the above construction gives types $\left(J, \lambda_{\Sigma}\right)$ which have the added property that if $(K, \tau)$ is an extension of $\left(J, \lambda_{\Sigma}\right)$ to the normalizer $K$ of $J$, a compact-mod-centre subgroup of $G$, then c- $\operatorname{Ind}_{K}^{G} \tau$ is an irreducible supercuspidal representation whose inertial class lies in $[G, \pi]_{G}$ Yu01].

Upon restriction to the pro- $p$ radical $H_{+}(\Sigma, G)$ of $H(\Sigma, G)$, the representation $\lambda_{\Sigma}$ is $\theta_{\Sigma}$-isotypic [HM08, Cor. 3.28], where

$$
\theta_{\Sigma}=\bigotimes_{i=0}^{d} \hat{\phi}^{i},
$$

understanding this product relative to the factorization (5.4). Following Ste05] we say that $\left(H_{+}(\Sigma, G), \theta_{\Sigma}\right)$ is the semisimple character associated to $\Sigma$. In the case that $\Sigma$ is supercuspidal, we say that $\theta_{\Sigma}$ is a simple character. These characters encode the arithmetic information contained within the type and will be essential to our arguments in Section 9 .

We conclude this section with a result relating data that differ only in the choice of $\left(J_{M}^{0}, \sigma\right)$, for some $M_{x, 0}^{0} \subseteq J_{M}^{0} \subseteq M_{x}^{0}$.

Recall that we write $J_{0}(\Sigma, G)=J(\Sigma, G) \cap G_{x, 0}$.

Lemma 5.7. The restriction of $\kappa_{\Sigma}$ to $J_{0}(\Sigma, G)$ is irreducible, and the irreducible components of $\left.\lambda_{\Sigma}\right|_{J_{0}(\Sigma, G)}$ are of the form $\sigma_{0} \otimes \kappa_{\Sigma}$, for some irreducible component $\sigma_{0}$ of $\left.\sigma\right|_{M_{x, 0}}$. Given some such irreducible component $\lambda_{0}=\sigma_{0} \otimes \kappa_{\Sigma}$, the pair $\left(J_{0}(\Sigma, G), \lambda_{0}\right)$ is an $\mathfrak{S}$-type, for some finite set $\mathfrak{S}$ of inertial equivalence classes, each of which is supported on $M$.

Proof. Let $\sigma_{0}$ be an irreducible component of $\left.\sigma\right|_{M_{x, 0}^{0}}$; then it is cuspidal and it follows from the definition that

$$
\Sigma_{0}=\left(\left(\overrightarrow{\mathbf{G}}, \mathbf{M}^{0}\right),(x,\{\iota\}),\left(M_{x, 0}^{0}, \sigma_{0}\right), \vec{r}, \vec{\phi}\right)
$$

is again a datum. Since $G_{x, 0}^{0} / G_{x, 0+}^{0} \cong M_{x, 0}^{0} / M_{x, 0+}^{0}$, we have for all $0 \leq i \leq d$ that $J^{i}\left(\Sigma_{0}, G\right)=J^{i}(\Sigma, G) \cap G_{x, 0}^{0}=J_{0}^{i}(\Sigma, G)$. Further, for each $0 \leq i<d$ we have $f^{i+1}\left(\Sigma_{0}, G\right)=J^{i+1}(\Sigma, G)$, whence the irreducible representations $\kappa_{0}^{i}$ (respectively, $\kappa^{i}$ ) of $J^{i+1}\left(\Sigma_{0}, G\right)$ (respectively, of $J^{i+1}(\Sigma, G)$ ) defined in (5.5) have the same degree. Thus $\kappa_{\Sigma}$ is an extension of $\kappa_{\Sigma_{0}}$, so restricts irreducibly; the second statement follows. Since $J^{d}\left(\Sigma_{0}, G\right)=J_{0}(\Sigma, G)$, the corresponding type is precisely $\left(J_{0}(\Sigma, G), \lambda_{0}\right)$, and thus the remaining statements follow from Proposition 5.6. 


\section{MaCKey TheORY AND UniCity CONJECTURES}

For the remainder of the paper we fix a supercuspidal datum $\Sigma=(\overrightarrow{\mathbf{G}}, x, \sigma, \vec{r}, \vec{\phi})$ for $G$ (with $J^{0}=G_{x}^{0}$ ) and freely use the additional terms defined in Definition 5.2 , Since $\Sigma$ is fixed, we omit it (and the pair $(\Sigma, G)$ ) from the notation for the most part. We also fix a choice of irreducible supercuspidal representation $\pi$ of $G$ such

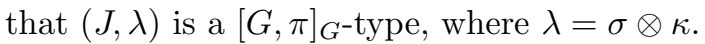

Our goal is to investigate Conjecture 6.1.

Conjecture 6.1 (The unicity of types). Suppose that $K$ is a maximal compact subgroup of $G$ and $(K, \tau)$ is a $[G, \pi]_{G}$-type. Then there exists a $g \in G$ such that ${ }^{g} J \subset K$ and $\operatorname{Hom}_{K}\left(\tau, \operatorname{Ind}_{g}^{K}{ }^{g} \lambda\right) \neq 0$.

Not every maximal compact subgroup $K$ will support a $[G, \pi]_{G}$-type $(K, \tau)$, but if it does, then $\tau$ must occur as an irreducible component of $\left.\pi\right|_{K}$.

Fix a point $y \in \mathscr{B}(G)$ such that $G_{y}$ is a maximal compact subgroup of $G$; every maximal compact subgroup of $G$ arises in this way. Since the restriction to $G_{y}$ of $\Pi:=\mathrm{c}-\operatorname{Ind}_{J}^{G} \lambda$ is a sum of countably many copies of $\left.\pi\right|_{G_{y}}$, our goal is equivalent to determining which, if any, of the irreducible components of $\left.\Pi\right|_{G_{y}}$ are $[G, \pi]_{G^{-} \text {types. }}$

We begin with the Mackey decomposition [Kut77]

$$
\left.\Pi\right|_{G_{y}}=\bigoplus_{g \in G_{y} \backslash G / J} \operatorname{Ind}_{g}^{G_{y}}{ }_{J \cap G_{y}}{ }^{g} \lambda .
$$

Instead of the component $\operatorname{Ind}_{g} G_{y \cap G_{y}}{ }^{g} \lambda$, we will prefer to consider its $g^{-1}$-conjugate

$$
\tau(y, g):=\operatorname{Ind}_{J \cap G_{g^{-1}}}^{G_{g-1}} \lambda .
$$

Note that, for a subrepresentation $\tau$ of $\tau(y, g)$, the pair $\left(G_{g^{-1} y}, \tau\right)$ is a $[G, \pi]_{G^{-}}$type if and only if $\left(G_{y},{ }^{g} \tau\right)$ is.

In Section 7 we will work instead with slightly larger representations, as follows. Fix an irreducible component $\sigma_{0}$ of $\left.\sigma\right|_{G_{x, 0}^{0}}$. Then $\Sigma_{0}=\left(\overrightarrow{\mathbf{G}}, x,\left(G_{x, 0}^{0}, \sigma_{0}\right), \vec{r}, \vec{\phi}\right)$ is again a supercuspidal datum. By Lemma 5.7 the corresponding pair $\left(J_{0}, \lambda_{0}\right)$ is an $\mathfrak{S}$-type for $\mathfrak{S}$ a finite set of inertial equivalence classes supported on $G$. Since $\lambda$ occurs as a subrepresentation of $\operatorname{Ind}_{J_{0}}^{J} \lambda_{0}$, the representation $\Pi_{0}:=\mathrm{c}-\operatorname{Ind}_{J_{0}}^{G} \lambda_{0}$ contains $\Pi$ as a direct summand. In particular, any type occuring in $\left.\Pi\right|_{G_{y}}$ also appears in $\left.\Pi_{0}\right|_{G_{y}}$.

More precisely, for each $g \in G_{y} \backslash G / J, \tau(y, g)$ occurs as a subrepresentation of

$$
\bigoplus_{h \in\left(J \cap G_{g^{-1} y}\right) \backslash J / J_{0}} \operatorname{Ind}_{J_{0} \cap G_{g^{-1}}}^{G_{g^{-1}}}{ }^{h} \lambda_{0},
$$

where these latter summands can variously be thought of as twisted Mackey components ${ }^{h} \tau_{0}(y, g h)$ of $\left.\Pi_{0}\right|_{G_{y}}$, or of twisted Mackey components of $\left.{ }^{h} \Pi_{0}\right|_{G_{y}}$.

These various decompositions into (twisted) Mackey components have a useful interpretation in terms of the building $\mathscr{B}(G)$. Namely, for each $g \in G, \tau(y, g)$ is a representation of the maximal compact subgroup $G_{g^{-1} y}$; we think of it as being attached to the point $g^{-1} y \in \mathscr{B}(G)$ (or, when convenient, to the point $\left[g^{-1} y\right] \in$ $\left.\mathscr{B}^{\text {red }}(G)\right)$. In fact, as $g$ ranges over a set of double coset representatives of $G_{y} \backslash G / J$, the representations are attached to distinct $J$-orbits of points in the $G$-orbit of $y$.

Recall that as $J$ is a compact open subgroup of $G$, the image $\mathscr{B}(G)^{J}$ in $\mathscr{B}^{\text {red }}(G)$ is a bounded and convex neighbourhood of $[x]$. The further that $\left[g^{-1} y\right]$ is from $[x]$ 
of the datum, the smaller is the intersection $J \cap G_{g^{-1} y}$, and thus the greater the likelihood that $\tau(y, g)$ (which depends only on $\left.\lambda\right|_{J \cap G_{g-1}}$ ) is contained in irreducible representations of $G$ lying in many different inertial equivalence classes.

In more precise terms, let $\left[x, g^{-1} y\right]$ denote the geodesic in $\mathscr{B}(G)$ from $x$ to $g^{-1} y$. Then $G_{x} \cap G_{g^{-1} y}=G_{\left[x, g^{-1} y\right]} \subset G_{z}$ for any $z \in\left[x, g^{-1} y\right]$. Since also $J \subset G_{x}$, we conclude that

$$
J \cap G_{g^{-1} y} \subset J \cap G_{z}
$$

for any $z \in\left[x, g^{-1} y\right]$.

We can say more. Associated to the datum $\Sigma$, we have a chain of generic embeddings $\mathscr{B}\left(G^{i}\right) \hookrightarrow \mathscr{B}\left(G^{i+1}\right)$, for $0 \leq i \leq d-1$; we use these to identify each building $\mathscr{B}\left(G^{i}\right)$ with a subspace of $\mathscr{B}(G)$. Recall that each of the buildings $\mathscr{B}(G)$ is a CAT $(0)$ space relative to the Bruhat-Tits metric; this means that for any point $z \in \mathscr{B}(G)$ one may find a unique closest point in $\mathscr{B}\left(G^{i}\right)$.

Definition 6.2. We let $\operatorname{proj}_{\mathscr{B}\left(G^{i}\right)}: \mathscr{B}(G) \rightarrow \mathscr{B}\left(G^{i}\right)$ denote the map which sends $z \in \mathscr{B}(G)$ to its unique closest point in $\mathscr{B}\left(G^{i}\right)$, relative to the fixed choice of embeddings.

While this map is algebraically a projection, it can interact with the geometric structure of buildings in a surprisingly delicate manner. For example, the restriction of $\operatorname{proj}_{\mathscr{B}\left(G^{i}\right)}$ to an apartment $\mathscr{A}$ of $\mathscr{B}(G)$ does not necessarily have image in $\mathscr{A}$. Nonetheless we have Lemma 6.3, which we'll use also in Section 8 to give a geometric interpretation of the key result in Section 7

Lemma 6.3. For any $z \in \mathscr{B}(G)$, and any $0 \leq i<d$, one has

$$
G_{z} \cap G^{i} \subset G_{\operatorname{proj}_{\mathscr{B}\left(G^{i}\right)}^{i}(z)} \cdot
$$

Proof. Let $d$ denote the Bruhat-Tits metric on $\mathscr{B}(G)$, and denote $z^{i}=\operatorname{proj}_{\mathscr{B}\left(G^{i}\right)}(z)$. Since each $g \in G^{i} \cap G_{z}$ fixes $z$ and acts by isometries on $\mathscr{B}(G)$, we have $d\left(z, g z^{i}\right)=$ $d\left(g z, g z^{i}\right)=d\left(z, z^{i}\right)$. Since $g \in G^{i}, g z^{i}$ is again in $\mathscr{B}\left(G^{i}\right)$, whence $g z^{i}=z^{i}$ by the uniqueness of $z^{i}$.

As an application, we have the following immediate result.

Proposition 6.4. Suppose that $g^{-1} y \in \mathscr{B}(G)^{J}$, the set of fixed points in $\mathscr{B}(G)$ under $J$. Then the pair $\left(G_{g^{-1} y}, \tau(y, g)\right)$ is a $[G, \pi]_{G}$-type.

Proof. Set $z=g^{-1} y$ and $\tau=\tau(y, g)$. By definition, we have $z \in \mathscr{B}(G)^{J}$ if and only if $J \subseteq G_{z}$. Let us show that $\tau=\operatorname{Ind}_{J}^{G_{z}} \lambda$ is irreducible; since it contains $\lambda$ upon restriction, it will follow that it is a $[G, \pi]_{G^{-}}$type. Let $z^{0}=\operatorname{proj}_{\mathscr{B}\left(G^{0}\right)}(z)$. Since $J=G_{x}^{0} G_{x, s_{0}}^{1} \cdots G_{x, s_{d-1}}^{d} \subset G_{z}$, we have by Lemma 6.3 that $G_{x}^{0} \subset G_{z} \cap G^{0} \subset G_{z^{0}}^{0}$. Since $x$ is a vertex of $\mathscr{B}\left(G^{0}\right)$, this implies $\left[z^{0}\right]=[x]$ and thus $G_{x}^{0}=G_{z^{0}}^{0}$.

A fundamental result of $\mathrm{Yu}$ Yu01, Corollary 15.5] implies that if $g \in G$ intertwines $\lambda$ (that is, satisfies $\operatorname{Hom}_{J \cap g}\left(\lambda,{ }^{g} \lambda\right) \neq 0$ ) then $g \in J G^{0} J$. Consequently, if $J \subset G_{z}$ and some $g \in G_{z}$ intertwines $\lambda$, we may conclude that $g \in J G^{0} J \cap G_{z}=J$. By Mackey theory, we conclude that $\operatorname{Ind}_{J}^{G_{z}} \lambda$ is irreducible.

Note that this fixed point set may meet the orbit of $[y]$ in 0,1 or finitely many points; this proposition implies that the corresponding Mackey components are irreducible. 
In fact, where Conjecture 6.1 only asserts that each type on a maximal compact subgroup can be constructed from a Yu type, we conjecture that in fact the $[G, \pi]_{G^{-}}$ typical representations of $G_{y}$ occuring in $\left.\Pi\right|_{G_{y}}$ are exactly the Mackey components corresponding to points in $G \cdot y \cap \mathscr{B}(G)^{J}$.

Conjecture 6.5. Suppose that $\tau(y, g)$ contains a $[G, \pi]_{G}$-type. Then $g^{-1} y \in$ $\mathscr{B}(G)^{J}$.

The methods of the next two sections are significant steps towards establishing this latter conjecture.

\section{MACKey COMPONENTS THAT INTERTWINE WITH NON-SUPERCUSPIDAL REPRESENTATIONS}

We continue to work with the supercuspidal datum $\Sigma=(\overrightarrow{\mathbf{G}}, x, \sigma, \vec{r}, \vec{\phi})$ fixed in Section [6, but focus on the S-type $\left(J_{0}, \lambda_{0}\right)=\left(J_{0}(\Sigma), \sigma_{0} \otimes \kappa_{\Sigma}\right)$.

Fix a Mackey component $\tau_{0}(y, g)$. Write $\mathrm{H}$ for the image of the map $J_{0} \cap G_{g^{-1} y} \rightarrow$ $J_{0} / J_{+}=G_{x, 0: 0+}^{0}$, and write $\mathrm{G}_{x}^{0}=G_{x, 0: 0+}^{0}$. In this section, we prove the following result, which is a significant generalization of [Lat17, Thm. 5.1].

Theorem 7.1. Suppose that there exists a proper parabolic subgroup $\mathrm{P}$ of $\mathrm{G}_{x}^{0}$ such that $\mathrm{H} \subset \mathrm{P}$. Then, for any irreducible component $\tau$ of $\tau_{0}(y, g)$, there exists a nonsupercuspidal irreducible representation $\pi^{\prime}$ of $G$ such that $\operatorname{Hom}_{G_{g^{-1}}}\left(\tau, \pi^{\prime}\right) \neq 0$. In particular, no irreducible component of $\tau_{0}(y, g)$ is a $\mathfrak{s}$-type for any $\mathfrak{s} \in \mathfrak{S}$.

The proof of this result will occupy the remainder of this section, taking the form of a series of lemmata. Fix a proper parabolic subgroup $\mathrm{P}$ of $\mathrm{G}_{x}^{0}$ containing $\mathrm{H}$, identified with a facet $\mathscr{F} \subset \mathscr{B}\left(G^{0}\right)$ adjacent to $x$. Choose a point $z \in \mathscr{F}$ for which P identifies with $G_{z, 0}^{0} / G_{x, 0+}^{0}$.

By MP96, $§ 6.3$, Proposition 6.4], we may associate to $z$ an $F$-Levi subgroup $\mathbf{M}^{0}$ of $\mathbf{G}^{0}$. Set $M^{0}=\mathbf{M}^{0}(F)$. For any 0-generic embedding $\mathscr{B}\left(M^{0}\right) \hookrightarrow \mathscr{B}\left(G^{0}\right)$, the point $z$ identifies with a vertex of $\mathscr{B}\left(M^{0}\right)$. The image of $M^{0} \cap G_{x, 0}^{0}$ in $G_{x, 0: 0+}^{0}$ is a Levi factor $\mathrm{M}$ of $\mathrm{P}$ that is isomorphic to $G_{z, 0: 0+}^{0}$ under the natural surjection. Write $\mathrm{P}=\mathrm{MN}$ for the resulting Levi decomposition of $\mathrm{P}$ in $\mathrm{G}_{x}^{0}$.

Choose an apartment $\mathscr{A}^{0}=\mathscr{A}\left(\mathbf{G}^{0}, \mathbf{S}^{0}, F\right) \subset \mathscr{B}\left(G^{0}\right)$ which contains both $x$ and $z$. Here, $\mathbf{S}^{0}$ is a maximal $F$-split torus in $\mathbf{G}^{0}$; let $\Phi^{0}=\Phi\left(\mathbf{G}^{0}, \mathbf{S}^{0}, F\right)$ be the corresponding rational root system. Then the point

$$
z^{\prime}:=x+(x-z) \in \mathscr{A}^{0}
$$

satisfies $\alpha\left(z^{\prime}-x\right)=-\alpha(z-x)$ for all $\alpha \in \Phi^{0}$; consequently, $G_{z^{\prime}, 0}^{0} / G_{x, 0+}^{0}=\overline{\mathrm{P}}$, the parabolic subgroup of $G_{x}^{0}$ which is opposite to $P$ relative to the Levi factor $M$. We have $M_{z, 0: 0+}^{0} \cong M_{z^{\prime}, 0: 0+}^{0} \cong \mathrm{M}$.

Decompose $\left.\sigma_{0}\right|_{\mathrm{M}}$ into irreducible components as

$$
\left.\sigma_{0}\right|_{\mathrm{M}}=\bigoplus_{\xi \in \Xi} \xi
$$

for $\Xi$ some multiset of irreducible representations of M. For each $\xi \in \Xi$, fix a representative $\left(\mathrm{L}_{\xi}, \zeta_{\xi}\right)$ of its cuspidal support; thus $\xi$ occurs as a summand of $\operatorname{Ind}_{\mathrm{L}_{\xi} \mathrm{U}_{\xi}}^{\mathrm{M}} \zeta_{\xi}$ for any parabolic $Q_{\xi}=L_{\xi} U_{\xi}$ of $M$ with Levi factor $L_{\xi}$.

Our first step is to show that for any pair $\left(L_{\xi}, \zeta_{\xi}\right)$ of Levi subgroup of $G_{x}^{0}$ and cuspidal representation $\zeta_{\xi}$, there is a natural corresponding deformation of the 
supercuspidal datum $\Sigma$ to a (non-supercuspidal) datum $\Sigma_{\xi}$, such that much of the structure of the corresponding types is well-related.

Lemma 7.2. There exists a point $u_{\xi} \in \mathscr{A}^{0}$ for which $G_{u_{\xi}, 0: 0+}^{0} \cong \mathrm{L}_{\xi}$, giving rise to a generalized twisted Levi sequence $\overrightarrow{\mathbf{L}}_{\xi}$ in $\mathbf{L}$ such that $\mathbf{L}_{\xi}^{i}$ is a Levi subgroup of $\mathbf{G}^{i}$ for each $0 \leq i \leq d$ and there is an $\vec{s}$-generic embedding $\iota$ of the corresponding buildings (relative to $u_{\xi}$ ) into $\mathscr{B}(G)$. Moreover, we have

$$
J_{\xi}:=G_{u_{\xi}, 0}^{0} G_{u_{\xi}, s_{0}}^{1} \cdots G_{u_{\xi}, s_{d-1}} \subset J_{0},
$$

where we have suppressed the notation $\iota$ on the left.

Proof. Since $\mathrm{L}_{\xi}$ is a Levi subgroup of $\mathrm{G}_{x}^{0}$ contained in $\mathrm{M}$, there exists a facet $\mathscr{F}_{\xi} \subset$ $\mathscr{A}^{0}$, whose closure contains both $x$ and $z^{\prime}$, such that for any $u_{\xi} \in \mathscr{F}_{\xi}$ we have $G_{u_{\xi}, 0: 0+}^{0} \cong \mathrm{L}_{\xi}$. More precisely, as above, associated to $u_{\xi}$ is a (proper) Levi subgroup $L_{\xi}^{0}=\mathbf{L}_{\xi}^{0}(F)$ of $G^{0}$. It has the property that with respect to any embedding of $\mathscr{B}\left(L_{\xi}^{0}\right)$ into $\mathscr{B}\left(G^{0}\right), u_{\xi}$ is a vertex of $\mathscr{B}\left(L_{\xi}^{0}\right)$ and the intersection $L_{\xi}^{0} \cap G_{u_{\xi}, 0}^{0}=\left(L_{\xi}^{0}\right)_{u_{\xi}, 0}$ is a maximal parahoric subgroup of $L_{\xi}^{0}$ whose image in $G_{x}^{0}$ is $L_{\xi}$. Now, given $\mathbf{L}_{\xi}^{0}$, apply the method of Kim and $\mathrm{Yu}$ (Remark 5.3) to construct a generalized twisted Levi sequence $\left(\mathbf{L}_{\xi}^{0}, \mathbf{L}_{\xi}^{1}, \ldots, \mathbf{L}_{\xi}^{d}=\mathbf{L}_{\xi}\right)$. We have that $\mathbf{L}_{\xi}^{i} \subseteq \mathbf{G}^{i}$ for each $i$ and $Z\left(\mathbf{L}_{\xi}^{0}\right) / Z\left(\mathbf{L}_{\xi}\right)$ is anisotropic. Set $L_{\xi}^{i}=\mathbf{L}_{\xi}^{i}(F)$.

We now choose a family of embeddings $\{\iota\}$ of the buildings $\mathscr{B}\left(L_{\xi}^{i}\right)$ into $\mathscr{B}\left(G^{i}\right)$ that is $\vec{s}$-generic, in the sense of [KY17, §3], and for which (7.2) holds as follows.

Since $Z\left(\mathbf{L}_{\xi}^{0}\right) / Z\left(\mathbf{L}_{\xi}\right)$ is anisotropic, $\mathscr{B}\left(L_{\xi}^{0}\right)$ embeds uniquely in $\mathscr{B}\left(L_{\xi}^{i}\right)$ for each $i$; this defines points $u_{\xi}$ in each of these buildings. By [KY17, Def 3.2], an embedding $\iota$ of $\mathscr{B}\left(L_{\xi}^{i}\right)$ into $\mathscr{B}\left(G^{i}\right)$ is $\left(u_{\xi}, s_{i-1}\right)$-generic if for each maximal split torus $\mathbf{S}^{i}$ of $\mathbf{L}_{\xi}^{i}$ such that $u_{\xi} \in \mathscr{A}\left(\mathbf{L}_{\xi}^{i}, \mathbf{S}^{i}\right)$ and, for each root $\alpha \in \Phi\left(\mathbf{G}^{i}, \mathbf{S}^{i}, F\right) \backslash \Phi\left(\mathbf{L}_{\xi}^{i}, \mathbf{S}^{i}, F\right)$, we have that $U_{\alpha, \iota\left(u_{\xi}\right), s_{i-1}}^{i}=U_{\alpha, \iota\left(u_{\xi}\right), s_{i-1}+}^{i}$; in particular, genericity is the condition that $\iota\left(u_{\xi}\right)$ does not lie on any of the hyperplanes $H_{\psi, s_{i-1}}^{i}=\left\{y \in \mathscr{A}\left(\mathbf{G}^{i}, \mathbf{S}^{i}, F\right) \mid\right.$ $\left.\psi(y)=s_{i-1}\right\}$, for any affine root $\psi$ with gradient such an $\alpha$.

As $i$ ranges from 0 to $d$, these hyperplanes $H_{\psi, s_{i-1}}^{i}$ subdivide each $\mathscr{B}\left(G^{i}\right) \subset$ $\mathscr{B}(G)$, leaving a collection $O^{i}$ of open connected components. We require a choice of embeddings $\iota: \mathscr{B}\left(L_{\xi}^{i}\right) \rightarrow \mathscr{B}\left(G^{i}\right)$ so that for each $i, \iota\left(u_{\xi}\right)$ lies in some $O^{i}$ that contains $x$ in its closure. This is possible, via the argument of [KY17, §3.6], since every $\alpha \in \Phi\left(\mathbf{G}^{i}, \mathbf{S}^{i}, F\right) \backslash \Phi\left(\mathbf{L}_{\xi}^{i}, \mathbf{S}^{i}, F\right)$ is non-constant on $\mathscr{F}_{\xi} \subset \mathscr{B}\left(G^{0}\right) \subset \mathscr{B}\left(G^{i}\right)$.

By construction, the geodesic $\left[x, \iota\left(u_{\xi}\right)\right]$ in $\mathscr{B}(G)$ does not cross any hyperplane $H_{\psi, s_{i-1}}$ in $\mathscr{B}\left(G^{i}\right)$ for any $i$, and so $U_{\alpha, \iota\left(u_{\xi}\right), s_{i-1}}^{i} \subset U_{\alpha, x, s_{i-1}}^{i}$ for all $\alpha \in$ $\Phi\left(\mathbf{G}^{i}, \mathbf{S}^{i}, F\right) \backslash \Phi\left(\mathbf{L}_{\xi}^{i}, \mathbf{S}^{i}, F\right)$; moreover, we have $U_{\alpha, u_{\xi}, s_{i-1}}^{i}=U_{\alpha, x, s_{i-1}}^{i}$ for each $\alpha \in$ $\Phi\left(\mathbf{L}_{\xi}^{i}, \mathbf{S}^{i}, F\right)$. This suffices to yield the inclusion $G_{\iota\left(u_{\xi}\right), s_{i-1}}^{i} \subset G_{x, s_{i-1}}^{i}$. Together, these inclusions imply (7.2).

With this choice of $\iota$ (which we fix once and for all, and omit from our notation), and letting $\zeta_{\xi}$ denote the inflation to $L_{\xi, 0}^{0}:=\left(L_{\xi}^{0}\right)_{u_{\xi}, 0}$ of the cuspidal representation of $L_{\xi}$ by the same name, we have the following.

Lemma 7.3. The tuple

$$
\Sigma_{\xi}:=\left(\left(\overrightarrow{\mathbf{G}}, \mathbf{L}_{\xi}^{0}\right),\left(u_{\xi},\{\iota\}\right),\left(L_{\xi, 0}^{0}, \zeta_{\xi}\right), \vec{r}, \vec{\phi}\right)
$$

is a datum. 
Proof. For each $i$, the character $\phi^{i}$ of $G^{i}$ is $G^{i+1}$-generic relative to $x$ and hence with respect to $u_{\xi} \in \mathscr{B}\left(G^{0}\right)$. The rest of the conditions from Definition [5.2 are immediate from the construction.

In particular, via $\Sigma_{\xi}$ we now associate to each irreducible component $\xi$ of $\left.\sigma_{0}\right|_{\mathrm{M}}$ a pair $\left(J_{\xi}, \lambda_{\xi}\right)$, where $J_{\xi}=J_{0}\left(\Sigma_{\xi}, G\right)$ as in (7.2) and $\lambda_{\xi}=\zeta_{\xi} \otimes \kappa_{\xi}$; here we write $\kappa_{\xi}=\left.\kappa\right|_{\Sigma_{\xi}}$. Note that $\left(J_{\xi}, \lambda_{\xi}\right)$ is an $\mathfrak{S}$-type for some finite set $\mathfrak{S}$ of inertial equivalence classes, all of which are supported on $L_{\xi}$; thus any irreducible subquotient of c-Ind $J_{\xi}^{G} \lambda_{\xi}$ is non-cuspidal. Thus Theorem 7.1 will follow if we show that, for any component $\tau$ of $\tau_{0}(y, g)$, there exists a $\xi \in \Xi$ such that $\tau$ is contained in an irreducible quotient of c-Ind $J_{\xi}^{G} \lambda_{\xi}$.

A first step, which will be needed in the proof, is to understand the image of $J_{\xi} \cap G_{g^{-1} y}$ in $\mathrm{G}_{x}^{0}$.

Lemma 7.4. For each $\xi \in \Xi$, the image $\mathrm{H}_{\xi}$ of $J_{\xi} \cap G_{g^{-1} y}$ in $\mathrm{G}_{x}^{0}$ is contained in a parabolic subgroup $\mathrm{Q}_{\xi}$ of $\mathrm{M}$ with Levi factor $\mathrm{L}_{\xi}$.

Proof. Since $J_{\xi} \subset J_{0}$, the image of $J_{\xi}$ lies in $\mathrm{G}_{x}^{0}$ via $J_{\xi} /\left(J_{\xi} \cap J_{+}\right) \cong J_{\xi} J_{+} / J_{+} \subset$ $J_{0} / J_{+} \cong \mathrm{G}_{x}^{0}$. Thus the image $\mathrm{H}_{\xi}$ of $J_{\xi} \cap G_{g^{-1} y}$ in $\mathrm{G}_{x}^{0}$ is a subgroup of the image $\mathrm{H}$ of $J_{0} \cap G_{g^{-1} y}$, which by hypothesis lies in the proper parabolic subgroup $\mathrm{P}$ of $\mathrm{G}_{x}^{0}$. On the other hand, we chose $u_{\xi}$ so that

$$
J_{\xi} /\left(J_{\xi} \cap J_{+}\right) \cong G_{u_{\xi}, 0}^{0} / G_{x, 0+}^{0} \subset G_{z^{\prime}, 0}^{0} / G_{x, 0+}^{0}=\overline{\mathrm{P}},
$$

the parabolic subgroup opposite to $\mathrm{P}$. Therefore $\mathrm{H}_{\xi} \subset \mathrm{M}=\mathrm{P} \cap \overline{\mathrm{P}}$. In fact, since $G_{u_{\xi}, 0}^{0} / G_{x, 0+}^{0}$ is itself a parabolic subgroup $\mathrm{P}^{\prime}$ of $\mathrm{G}_{x}^{0}$ with Levi factor $\mathrm{L}_{\xi}$, we can set $Q_{\xi}=P^{\prime} \cap M$, which is a parabolic subgroup of $M$ with Levi decomposition $\mathrm{Q}_{\xi}=\mathrm{L}_{\xi} \mathrm{N}_{\xi}$, and deduce further that

$$
\mathrm{H}_{\xi} \subset \mathrm{L}_{\xi} \mathrm{N}_{\xi}=\mathrm{Q}_{\xi} \subset \mathrm{M},
$$

as desired.

The crucial technical step is for us to compare the Heisenberg-Weil representations $\kappa$ and $\kappa_{\xi}$.

Proposition 7.5. The restriction of $\kappa$ to $J_{\xi}$ is $\kappa_{\xi}$-isotypic.

We will need the following basic lemma about Heisenberg representations.

Lemma 7.6. Suppose $\mathcal{H}_{1} \subset \mathcal{H}_{2}$ are finite Heisenberg p-groups with common centre z. Let $\phi$ be a non-trivial character of $\mathcal{Z}$, and let $\eta_{1}, \eta_{2}$ be irreducible representations of $\mathcal{H}_{1}, \mathcal{H}_{2}$, respectively, both with central character $\phi$. Then $\left.\eta_{2}\right|_{\mathcal{H}_{1}} \simeq \eta_{1}^{\oplus\left[\mathcal{H}_{2}: \mathcal{H}_{1}\right]^{1 / 2}}$.

Proof. By the Stone-von Neumann theorem, the irreducible representations of a finite Heisenberg $p$-group $\mathcal{H}_{i}$ with centre $z$ are either characters or $\left[\mathcal{H}_{i}: z\right]^{1 / 2}$ dimensional, and the latter are uniquely determined by their non-trivial central characters.

Proof of Proposition 7.5. Recall that $\kappa$ is a tensor product $\kappa_{0} \otimes \kappa_{1} \otimes \cdots \otimes \kappa_{d}$, where each $\kappa^{i}$ is obtained from the character $\phi^{i}$ via a Heisenberg-Weil lift (and $\kappa_{d}=\phi^{d}$ ). We wish to compare the constructions of the representations $\kappa_{i}$ and $\kappa_{\xi, i}$ for each $0 \leq i \leq d-1$. Fix an index $i$ throughout. 
The essential difference between the groups $J$ and $J_{\xi}$ is the replacement of the point $x$ with $u=u_{\xi}$. We therefore write $J_{x}=J$ and $J_{u}=J_{\xi}$ in order to keep track of and further emphasize this distinction. For each $w \in\{u, x\}$, we have the groups

$$
\partial_{w}^{i+1}=\left(G^{i}, G^{i+1}\right)_{w,\left(r_{i}, s_{i}\right)}, \text { and } \partial_{w+}^{i+1}=\left(G^{i}, G^{i+1}\right)_{w,\left(r_{i}, s_{i}+\right)} .
$$

Recall that we extend the character $\left.\phi^{i}\right|_{G_{x, r_{i}}^{i}}$ to a character $\hat{\phi}_{w}^{i}$ of $j_{w+}^{i+1}$ and write $\mathcal{N}_{w}^{i+1}=\operatorname{ker} \hat{\phi}_{w}^{i}$. We further define

$$
\begin{aligned}
\mathcal{H}_{w} & =\mathcal{J}_{w}^{i+1} / \mathcal{N}_{w}^{i+1}, \\
\mathcal{W}_{w} & =\mathcal{J}_{w}^{i+1} / \mathcal{J}_{w+1}^{i+1}, \text { and } \\
\mathcal{Z}_{w} & =\mathcal{J}_{w+}^{i+1} / \mathcal{N}_{w}^{i+1} .
\end{aligned}
$$

By [HM08, $\S 3.3]$, the character $\hat{\phi}_{w}^{i}$ of $\partial_{w+}^{i+1}$ is characterized by the property that it coincides with $\phi^{i}$ on $G_{w, r_{i}}^{i}$, and is trivial on $\left(G^{i}, G^{i+1}\right)_{w,\left(r_{i}+, s_{i}+\right)}$. Thus, in particular, $G_{w, r_{i}}^{i} \mathcal{N}_{w}^{i+1}=\mathrm{J}_{w+}^{i+1}$, whence

$$
\mathrm{j}_{w+}^{i+1} / \mathcal{N}_{w}^{i+1} \simeq G_{w, r_{i}}^{i} / \operatorname{ker} \phi^{i} .
$$

Fix a minimal Levi subgroup $C^{i}$ of $G^{i}$, arising as the centralizer of a maximal split torus $S^{i}=\mathbf{S}^{i}(F)$ such that both $u$ and $x$ are contained in the apartment $\mathscr{A}\left(\mathbf{G}^{i}, \mathbf{S}^{i}, F\right)$. Then, as noted in Remark 3.2, the Moy-Prasad filtration on $C^{i}$ is independent of the choice of $w \in\{u, x\}$. Since the fundamental group of the derived group $\mathbf{G}_{\mathrm{der}}^{i}$ is a subgroup of the fundamental group of $\mathbf{G}_{\mathrm{der}}$, and $p$ is coprime to the order of the latter, we may apply Kal19, Lemma 3.5.1] to infer that since $\phi^{i}$ is a character of $G^{i}$, it is trivial on $\mathbf{G}_{\text {der }}^{i}(F)_{x, 0+}$. In particular, $\phi^{i}$ is trivial on the root subgroups of $G^{i}$ at depth $r_{i}$, which implies that

$$
G_{w, r_{i}}^{i} / \operatorname{ker} \phi^{i} \simeq C_{r_{i}}^{i} / \operatorname{ker} \phi^{i} .
$$

This allows us to identify $z_{u}$ with $z_{x}$ and, moreover, allows us to observe that the restrictions of $\hat{\phi}_{u}^{i}$ and $\hat{\phi}_{x}^{i}$ coincide with that of $\phi^{i}$ under this identification. Thus for each $w \in\{u, x\}$, the character $\left.\phi^{i}\right|_{C_{r_{i}}^{i}}$ defines a symplectic structure on $\mathcal{W}_{w}$, and the structure of a Heisenberg $p$-group on $\mathcal{H}_{w}$, with centre $\mathcal{Z}_{w}$. Our first step is to show that $\mathcal{W}_{u}$ is a symplectic subspace of $\mathcal{W}_{x}$.

Begin by considering our Levi subgroups $L^{j}:=L_{\xi}^{j}$ for $j \in\{i, i+1\}$. Since $L_{x, r}=L_{u, r}$ for all $r \geq 0$ by construction of $L$, the following groups are in fact independent of the choice of $w \in\{u, x\}$

$$
\mathcal{J}^{L}=\left(L^{i}, L^{i+1}\right)_{w,\left(r_{i}, s_{i}\right)} \text { and } \mathcal{J}_{+}^{L}=\left(L^{i}, L^{i+1}\right)_{w,\left(r_{i}, s_{i}+\right)} .
$$

Set $\mathcal{W}^{L}=\mathcal{J}^{L} / \mathcal{J}_{+}^{L}$. The genericity of the embedding of $\mathscr{B}\left(L^{i}\right)$ into $\mathscr{B}\left(G^{i}\right)$ directly implies that $\mathcal{W}_{u} \cong \mathcal{W}^{L}$, as in the proof of [KY17, Thm 7.5]. Because the root subgroups of $L^{i+1}$ are root subgroups of $G^{i+1}$, we deduce that $\mathcal{W}^{L}$ is a subspace of $\mathcal{W}_{x}$; the compatibility of their symplectic forms then implies that $\mathcal{W}_{u}$ is a symplectic subspace of $\mathcal{W}_{x}$.

The inclusion of $\mathcal{J}^{L}$ into $\mathcal{J}_{x}^{i+1}$ thus induces an injective homomorphism $i: \mathcal{H}_{u} \hookrightarrow$ $\mathcal{H}_{x}$, which restricts to an isomorphism of the centres of these Heisenberg $p$-groups. Let $\left(\eta_{w}, V_{w}\right)$ denote the Heisenberg representation of $\mathcal{H}_{w}$ with central character $\phi^{i}$. By Lemma 7.6. $\eta_{x}$ becomes $\eta_{u}$-isotypic upon restriction to $\mathcal{H}_{u}$. 
Now consider the Weil lifts of each of these representations. For $w \in\{u, x\}$, they are homomorphisms

$$
\hat{\eta}_{w}: \operatorname{Sp}\left(\mathcal{W}_{w}\right) \ltimes \mathcal{H}_{w} \rightarrow \operatorname{Aut}\left(V_{w}\right) .
$$

Note that $\hat{\eta}_{w}$ is characterized as the unique representation of $\operatorname{Sp}\left(\mathcal{W}_{w}\right) \ltimes \mathcal{H}_{w}$ extending $\eta_{w}$ [HM08, $\left.\S 2.3\right]$. (When $p=3$ and $\operatorname{dim}_{\mathbb{F}_{p}}\left(\mathcal{W}_{w}\right)=2$, there are two choices and we take the one associated to the central character of $\eta_{w}$ [HM08, §2.4].) The restriction of $\hat{\eta}_{x}$ to the subgroup $\operatorname{Sp}\left(\mathcal{W}_{u}\right) \ltimes \mathcal{H}_{u}$, being $\eta_{u}$-isotypic upon further restriction to $\mathcal{H}_{u}$, must therefore be $\hat{\eta}_{u}$-isotypic.

Finally, the representation $\kappa_{w, i}$ of $J_{w}^{i+1}$ is obtained from $\phi^{i}$ and $\hat{\eta}_{w}$ by making the identification

$$
J_{w}^{i+1}=G_{w, 0}^{0} G_{w, s_{0}}^{1} \cdots G_{w, s_{i}}^{i+1}=J_{w}^{i} \partial_{w}^{i+1}
$$

and then, for all $g \in J_{w}^{i}$ and all $j \in \mathcal{J}_{w}^{i+1}$, setting

$$
\kappa_{w, i}(g j)=\phi^{i}(g) \hat{\eta}_{w}(g, j) .
$$

Thus, upon restriction, the representation $\kappa_{x, i}$ is $\kappa_{u, i}$-isotypic, and the same is true of their respective inflations to $J_{0}=J_{0}^{d}(\Sigma, G)$ and $J_{\xi}=J_{0}^{d}\left(\Sigma_{\xi}, G\right)$.

With this, we are ready to complete the proof.

Proof of Theorem 7.1. Let $\tau$ be an irreducible component of $\tau_{0}(y, g)=\operatorname{Ind}_{J_{0} \cap G_{g}-1 y}^{G_{g}-1_{y}} \lambda_{0}$, where $\lambda_{0}=\sigma_{0} \otimes \kappa$. Frobenius reciprocity implies $\operatorname{Hom}_{J_{0} \cap G_{g^{-1}}}\left(\tau, \sigma_{0} \otimes \kappa\right) \neq 0$. We therefore have non-trivial intertwining between these representations on the smaller subgroup $J_{0} \cap G_{g^{-1} y} \cap M_{x, 0}^{0} G_{x, 0+}$, whose image in $\mathrm{G}_{x}^{0}$ lies in M. Since $\left.\sigma_{0}\right|_{\mathrm{M}}=\oplus_{\xi \in \Xi} \xi$, we may choose $\xi$ for which

$$
\operatorname{Hom}_{J_{0} \cap G_{g^{-1}} \cap} \cap M_{x, 0}^{0} G_{x, 0+}(\tau, \xi \otimes \kappa) \neq 0,
$$

where here we think of $\xi$ as a representation of $M_{x, 0}^{0} G_{x, 0+}$ by inflation. By Lemmas 7.2 and 7.4 , we have

$$
J_{\xi} \cap G_{g^{-1} y} \subset J_{0} \cap G_{g^{-1} y} \cap M_{x, 0}^{0} G_{x, 0+} .
$$

Moreover, by Proposition 7.5 the restriction to $J_{\xi} \cap G_{g^{-1} y}$ of $\kappa$ is $\kappa_{\xi}$-isotypic. Therefore we may further conclude that

$$
\operatorname{Hom}_{J_{\xi} \cap G_{g^{-1}}}\left(\tau, \xi \otimes \kappa_{\xi}\right) \neq 0 .
$$

The cuspidal support of $\xi$ is $\left(\mathrm{L}_{\xi}, \zeta_{\xi}\right)$; thus choosing the parabolic $\mathrm{Q}_{\xi}$ of M with Levi factor $L_{\xi}$ as in Lemma 7.4, we have

$$
0 \neq \operatorname{Hom}_{\mathrm{M}}\left(\xi, \operatorname{Ind}_{Q_{\xi}}^{\mathrm{M}} \zeta_{\xi} \otimes 1\right)=\operatorname{Hom}_{Q_{\xi}}\left(\xi, \zeta_{\xi} \otimes 1\right),
$$

with the latter identification following by Frobenius reciprocity.

Now consider the datum $\Sigma_{\xi}$ of Lemma 7.3. It follows from Lemma 7.4 that

$$
\operatorname{Hom}_{J_{\xi} \cap G_{g^{-1}}}\left(\tau, \zeta_{\xi} \otimes \kappa_{\xi}\right) \neq 0 .
$$

Since $\tau$ is irreducible as a representation of $G_{g^{-1} y}$, applying Frobenius reciprocity reveals that $\tau$ is a subrepresentation of

$$
\pi_{\xi}:=\operatorname{Ind}_{J_{\xi} \cap G_{g^{-1}}}^{G_{g^{-1}}} \zeta_{\xi} \otimes \kappa_{\xi} .
$$

Note that by Mackey theory, we have

$$
\operatorname{Res}_{G_{g^{-1}}}^{G}{ }^{\mathrm{c}-\operatorname{Ind}_{J_{\xi}}^{G} \zeta_{\xi} \otimes \kappa_{\xi}=} \bigoplus_{h \in G_{g^{-1} y} \backslash G / J_{\xi}} \operatorname{Ind}_{h}^{G_{J^{-1}} \cap G_{g^{-1}}}{ }^{h}\left(\zeta_{\xi} \otimes \kappa_{\xi}\right) .
$$


The summand for $h=1$ is exactly the representation $\pi_{\xi}$. It follows that $\tau$ is contained in c-Ind $J_{\xi}^{G} \zeta_{\xi} \otimes \kappa_{\xi}$, and hence, by Lemma 4.2 in some finitely generated subquotient, which admits an irreducible quotient $\pi^{\prime}$ containing $\tau$. Since $\left(J_{\xi}, \zeta_{\xi} \otimes\right.$ $\left.\kappa_{\xi}\right)$ is an $\mathfrak{S}$-type for a set of non-cuspidal inertial classes $\mathfrak{S}$ supported on $L_{\xi}$ ( $c f$. Lemma 5.7), this representation $\pi^{\prime}$ is non-cuspidal. Consequently, $\left(G_{g^{-1} y}, \tau\right)$ is not a $[G, \pi]_{G^{-t y p e}}$

Remark 7.7. The construction carried out during this section makes use of only those representation-theoretic properties inherited by $\tau$ from the Mackey decomposition of $\pi$.

\section{Projection maps Associated to twisted Levi SubGroups}

In this section, we give a geometric interpretation of the hypothesis of Theorem 7.1 and illustrate these ideas with an example.

For each $x \in \mathscr{B}(G)$, write $\widetilde{[x]}=\{[x]\} \times X_{*}(Z(\mathbf{G})) \otimes_{\mathbb{Z}} \mathbb{R}$ for the corresponding fibre over $[x] \in \mathscr{B}^{\text {red }}(G)$. For a point $z \in \mathscr{B}(G)$, the group $G_{x} \cap G_{z}$ fixes both $x$ and $z$, as well as every element $x^{\prime}$ of $\widetilde{[x]}$; from this we see that $G_{x} \cap G_{z}$ also fixes each of the geodesics from $\widetilde{[x]}$ to $\widetilde{[z]}$. It may also fix more, such as if any of these geodesics pass through the interior of a chamber. We set $\Gamma(x, z)=\mathscr{B}(G)^{G_{x} \cap G_{z}}$ for the full set of fixed points.

Proposition 8.1. Let $\mathrm{H}_{z}$ denote the image of the map $J_{0} \cap G_{z} \rightarrow J_{0} / J_{+}=G_{x, 0: 0+}^{0}$, and write $\mathrm{G}_{x}^{0}=G_{x, 0: 0+}^{0}$. Suppose that $\operatorname{proj}_{\mathscr{B}\left(G^{0}\right)} \Gamma(x, z) \neq \widetilde{[x]}$. Then $\mathrm{H}_{z}$ is contained in a proper parabolic subgroup of $\mathrm{G}_{x}^{0}$.

Proof. Suppose that $\operatorname{proj}_{\mathscr{B}\left(G^{0}\right)} \Gamma(x, z)$ is strictly larger than $\widetilde{[x]}$; then it meets a facet $\mathscr{F}$ of $\mathscr{B}\left(G^{0}\right)$ adjacent to $x$. For any $z^{0} \in \mathscr{F}$, the group $\mathrm{P}=G_{z^{0}, 0}^{0} / G_{x, 0+}^{0}$ is a proper parabolic subgroup of $\mathrm{G}_{x}^{0}$.

So choose $z^{0} \in \mathscr{F}$ lying in the image of the projection of some point $z^{\prime} \in \Gamma(x, z)$. Then $J \cap G_{z} \subset G_{x} \cap G_{z}=G_{\Gamma(x, z)} \subset G_{z^{\prime}}$. Moreover, the image $\mathrm{H}_{z}$ of $J_{0} \cap G_{z}$ in $J_{0} / J_{+}$is contained in the image of $G_{x, 0}^{0} \cap G_{z^{\prime}}$. By Lemma 6.3, $G^{0} \cap G_{z^{\prime}} \subset G_{z^{0}}^{0}$; intersecting further with the kernel of the Kottwitz homomorphism, we deduce that $G_{x, 0}^{0} \cap G_{z^{\prime}} \subset G_{z^{0}, 0}^{0}$, as required.

We deduce the following corollary of Theorem 7.1 .

Corollary 8.2. If

$$
\operatorname{proj}_{\mathscr{B}\left(G^{0}\right)} \Gamma\left(x, g^{-1} y\right) \neq \widetilde{[x]},
$$

then $\tau(y, g)$ does not contain any $[G, \pi]_{G}$-typical representations.

Let

$$
\Delta=\operatorname{proj}_{\mathscr{B}\left(G^{0}\right)}^{-1}(\widetilde{[x]})
$$

be the set of points in $\mathscr{B}(G)$ whose projection to $\mathscr{B}\left(G^{0}\right)$ lies in $\widetilde{[x]}$. A sufficient condition for $z=g^{-1} y$ to satisfy the hypothesis of Theorem 7.1 is that $z \notin \Delta$, but this condition is not necessary. In fact, there are many points $z \in \Delta$ for which the group $\mathrm{H}_{z}$ is contained in some proper parabolic subgroup of $\mathrm{G}_{x}^{0}$. We illustrate this in Example 8.5 in the case of $\mathbf{G}=\mathbf{S p}_{4}$. 
First, however, let us describe some sets of points in $\Delta$ where Theorem 7.1 cannot apply - among them, the points on the buildings of suitably complementary twisted Levi subgroups.

Since $\Sigma$ is a supercuspidal datum, the connected component $\left(\mathbf{Z}^{0}\right)^{\circ}$ of the identity of the centre $\mathbf{Z}^{0}$ of $\mathbf{G}^{0}$ is a $G$-minisotropic (that is, anisotropic $\bmod Z(\mathbf{G})$ ) torus of $\mathbf{G}^{0}$; moreover $\mathbf{G}^{0}=\operatorname{Cent}_{\mathbf{G}^{0}}\left(\mathbf{Z}^{0}\right)$. We set $Z^{0}=\mathbf{Z}^{0}(F)$, and write $Z_{\mathrm{b}}^{0}$ for its maximal bounded subgroup.

Definition 8.3. Let $\tilde{Z}^{0}=\tilde{\mathbf{Z}}^{0}(F)$ be a $G$-minisotropic torus contained in $G^{0}$ such that $\tilde{Z}_{\mathrm{b}}^{0} \subset G_{x}^{0}$, and such that if $T=\left\langle Z^{0}, \tilde{Z}^{0}\right\rangle$, then $\mathrm{T}:=T_{0} / T_{0+}$ is a minisotropic maximal torus of $\mathrm{G}_{x}^{0}$. Set $\tilde{\mathbf{G}}^{0}=\operatorname{Cent}_{\mathbf{G}}\left(\tilde{\mathbf{Z}}^{0}\right)$, which is a twisted Levi subgroup of G. We call $\tilde{G}^{0}=\tilde{\mathbf{G}}^{0}(F)$ a complementary twisted Levi subgroup (of $G$ to $G^{0}$ at $x$ ).

As one example, one may choose for $\tilde{Z}^{0}$ an unramified minisotropic torus such that $\tilde{Z}_{0: 0+}^{0}$ is a maximal torus of $\mathrm{G}_{x}^{0}$. However, there exist many more examples, corresponding to ramified tori.

Given such a complementary twisted Levi subgroup, any embedding $\mathscr{B}\left(\tilde{G}^{0}\right) \hookrightarrow$ $\mathscr{B}(G)$ has image containing $x$ (and in fact all of $\widetilde{[x]}$ ) and is contained in $\mathscr{B}(G)^{\tilde{Z}_{0}^{0}}$.

Proposition 8.4. Let $\tilde{Z}^{0}$ be any torus as in Definition 8.3 . Then $\mathscr{B}(G)^{\tilde{Z}_{0}^{0}} \subseteq \Delta$ and for any $z \in \mathscr{B}(G)^{\tilde{Z}_{0}^{0}}$, the group $\mathrm{H}_{z}$ is not contained in any proper parabolic subgroup of $\mathrm{G}_{x}^{0}$.

Proof. Since $z$ is fixed by $\tilde{Z}_{0}^{0}$ and by construction of $\tilde{Z}_{0}^{0} \subset G_{x, 0}^{0}$, we have $\tilde{Z}_{0}^{0} \subset$ $G_{z} \cap G_{x, 0}^{0}$, and hence $\tilde{Z}_{0: 0+}^{0} \subset \mathrm{H}_{z}$. If $\mathrm{P}$ is a parabolic subgroup of $\mathrm{G}_{x}^{0}$ containing $\mathrm{H}_{z}$, then it further contains $Z_{0: 0+}^{0}$, hence the minisotropic maximal torus $\mathrm{T}$. We conclude that $\mathrm{P}=\mathrm{G}_{x}^{0}$. By Proposition 8.1 if follows that $\mathscr{B}(G)^{\tilde{Z}_{0}^{0}} \subset \Delta$.

Consequently, there are infinitely many points $z=g^{-1} y$ in $\mathscr{B}(G)$, and corresponding twisted Mackey components, to which Theorem 7.1 will not apply; we address some of these in the next section.

We now provide an example which illustrates the complexity of the fibre $\Delta$ of the projection map over $\widetilde{[x]}$. In particular, we exhibit points $z \in \Delta$ to which each of Propositions 8.1 and 8.4 apply.

Example 8.5. Let $G=\mathbf{S p}_{4}(F)$ and let $y$ be a vertex of $\mathscr{B}(G)$. Choose a twisted Levi sequence of $\left(G^{0}, G\right)$ of length two, where $G^{0} \cong Z^{0} \times \mathbf{S L}_{2}(F)$ with $Z^{0}=\mathbf{Z}^{0}(F)$ an unramified $F$-anisotropic torus of rank 1. Suppose the vertex $x \in \mathscr{B}\left(G^{0}\right)$ maps to a hyperspecial vertex of $\mathscr{B}(G)$. The rest of the datum is fully specified by a choice of character $\chi$ of $Z^{0}$ of depth $r(=2 s)$, and a cuspidal representation $\sigma$ of $\mathbf{S L}_{2}(\mathfrak{f})$. Let $\mathfrak{s}$ be the inertial class of the corresponding irreducible supercuspidal representation of $G$. Note that here, $J=G_{x}^{0} G_{x, s}=J_{0}$ and, since $G^{0}$ is unramified, $\mathscr{B}(G)^{J}=\{x\}$.

We first consider the link of $x$ in $\mathscr{B}(G)$, which is the closure $\Omega(x, 1)$ of all chambers adjacent to $x$. As all geodesics $\left[x, g^{-1} y\right]$ meet $\Omega(x, 1)$, the goal is to classify the points in $\Omega(x, 1)$ to which Theorem 7.1 applies.

Let $\mathscr{A}$ be an apartment containing $x$. Set $\Omega_{\mathscr{A}}=\Omega(x, 1) \cap \mathscr{A}$ and $\Omega_{\mathscr{A}}^{0}=\Omega_{\mathscr{A}} \cap$ $\mathscr{B}\left(G^{0}\right)$. Then $\Omega_{\mathscr{A}}^{0}$ contains $x$, as well as up to two chambers of $\mathscr{B}\left(G^{0}\right)$. We illustrate some possible configurations in Figure 8.1.

In the simplest case, $\Omega_{\mathscr{A}}^{0}$ contains two chambers of $\mathscr{B}\left(G^{0}\right)$, and then the restriction to $\Omega_{\mathscr{A}}$ of $\operatorname{proj}_{\mathscr{B}\left(G^{0}\right)}$ coincides with the orthogonal projection map. Thus 

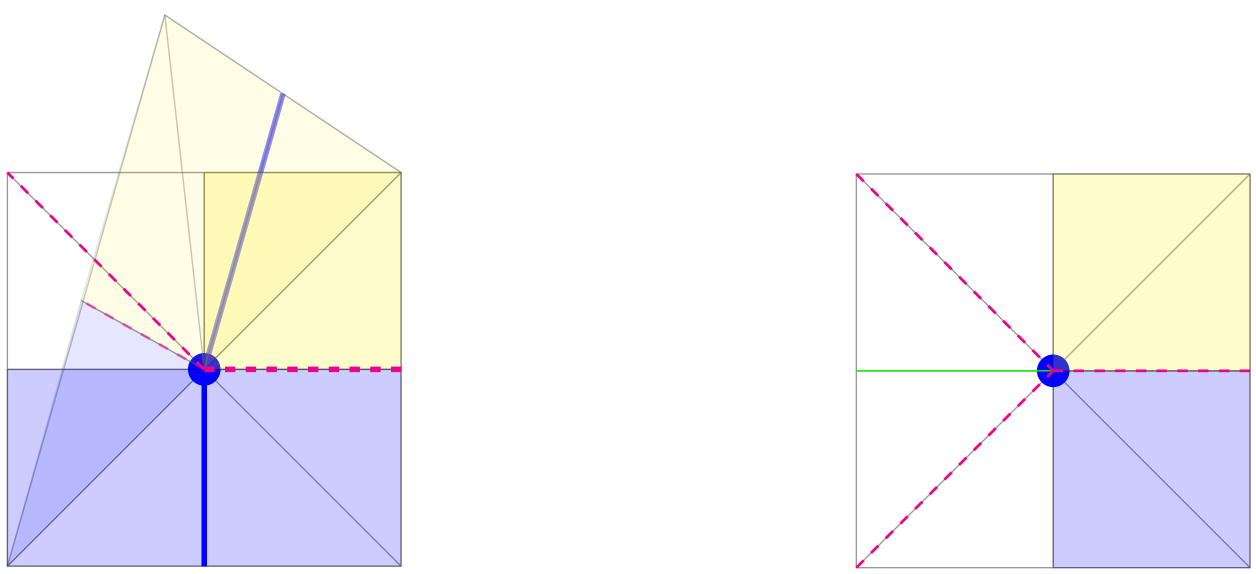

Figure 8.1. Neighbourhoods of $x$ in three apartments. The dark blue line represents their intersection with $\mathscr{B}\left(G^{0}\right)$. Chambers of $\Delta=\operatorname{proj}_{\mathscr{B}\left(G^{0}\right)}^{-1}(\widetilde{[x]})$ are white and for all complementary twisted Levis $\tilde{G}^{0}$, the intersection of these apartments with $\mathscr{B}\left(\tilde{G}^{0}\right)=\mathscr{B}(G)^{\tilde{Z}_{0}^{0}}$ is indicated by magenta dashed lines. The points whose projection onto $\mathscr{B}\left(G^{0}\right)$ lies in some chamber of $\mathscr{B}\left(G^{0}\right)$ are indicated by colours: blue, yellow, and green, corresponding to distinct chambers of $\mathscr{B}\left(G^{0}\right)$ (not all shown).

Proposition 8.1 applies to all points save those on the orthogonal complement of $\Omega_{\mathscr{A}}^{0}$ through $x$, which is $\Delta \cap \mathscr{A}$. (This set coincides with $\Omega_{\mathscr{A}} \cap \mathscr{B}\left(\tilde{G}^{0}\right)$, where $\tilde{G}^{0}=\mathbf{S L}_{2}(F) \times Z^{0}$ is a complementary twisted Levi.) Suppose $\left[x, g^{-1} y\right]$ meets a point $z$ of $\Delta \cap \mathscr{A}$ in a facet adjacent to $x$; then we compute $G_{g^{-1}} \cap J \subseteq G_{z} \cap J \subseteq$ $Z_{0+}^{0} \times \mathbf{S L}_{2}(\mathfrak{o})$. Thus by multiplying $\chi$ by a depth-zero character of $Z^{0}$ we obtain the datum of a new supercuspidal representation containing ${ }^{g} \tau(y, g)$, whence this latter cannot contain an $\mathfrak{s}$-type. We conclude that if $g^{-1} y \in \mathscr{A}$ then $\tau(y, g)$ contains an $\mathfrak{s}$-type if and only if $g^{-1} y=x$.

When $\Omega_{\mathscr{A}}^{0}$ contains only one chamber $\mathscr{F}$ of $\mathscr{B}\left(G^{0}\right)$ the geometry becomes more complex. Let $H$ denote the part of $\Omega_{\mathscr{A}}$ which is the union of lines which meet $\mathscr{F}$ at a right angle; since these are geodesics, we conclude they represent the projection onto $\mathscr{B}\left(G^{0}\right)$. For other points $z \in \Omega_{\mathscr{A}}$, however, even if the nearest point in $\Omega_{\mathscr{A}}^{0}$ is $x$, then it need not follow that $x=\operatorname{proj}_{\mathscr{B}\left(G^{0}\right)}(z)$ - it may be that there is a closer point in $\mathscr{B}\left(G^{0}\right)$ to $z$ which lies in a different apartment. We illustrate this phenomenon in the first figure of Figure 8.1, where the two overlapping apartments depicted differ by conjugation by an element of the appropriate root subgroup. One sees that $\Delta$ contains chambers of $\mathscr{B}(G)$, in addition to rays corresponding to buildings of twisted Levi subgroups as in Proposition 8.1

Finally, when $\Omega_{\mathscr{A}}^{0}=\{x\}$, no properties of the map $\operatorname{proj}_{\mathscr{B}\left(G^{0}\right)}$ may be inferred from $\mathscr{A}$. As illustrated in the second figure of Figure 8.1 , some chambers of $\Omega_{\mathscr{A}}$ will lie in $\Delta$, but not all.

In fact, in all of the examples illustrated in Figure 8.1, it can be shown that all chambers of $\Omega_{\mathscr{A}}$ satisfy the hypotheses of Theorem [7.1 with those $z$ lying in chambers of $\Delta$ yielding particularly small subgroups $\mathrm{H}$. The only points in these 
examples excluded from the application of Theorem 7.1 are those of $\mathscr{B}\left(\tilde{G}^{0}\right)$, for various complementary twisted Levi subgroups $\tilde{G}^{0}$.

\section{A result about simple characters}

In this section, we establish another condition on $g^{-1} y$ which suffices to show that the twisted Mackey component $\tau(y, g)$ does not contain any $[G, \pi]_{G}$-typical representations. The proof is a generalization of [Pas05, Prop. 7.2] to this general setting. We then derive a geometric interpretation of its hypothesis, to illustrate how the method conjecturally applies to the points $g^{-1} y$ outside a small neighbourhood of $x$ and lying in the subset $\Delta$ constructed in Section 5 .

We continue to work with the supercuspidal datum $\Sigma=(\overrightarrow{\mathbf{G}}, x, \sigma, \vec{r}, \vec{\phi})$ from Section 6. In particular, recall that the subgroup $H_{+}=G_{x, 0+}^{0} G_{x, s_{0}+}^{1} \cdots G_{x, s_{d-1}+}^{d}$ of $J=G_{x}^{0} G_{x, s_{0}}^{1} \cdots G_{x, s_{d-1}}^{d}$ carries the simple character $\theta_{\Sigma}$, obtained from the sequence $\vec{\phi}$ by an appropriate inflation-restriction process, as outlined in Section 5 .

Lemma 9.1. The subgroup $H_{+}$of $J$ is normal; hence for each $t>0$ and $1 \leq i \leq d$, $J^{i}$ normalizes $H_{t}=H_{+} \cap G_{x, t}$.

Proof. Since $J^{i}=J \cap G^{i} \subset G_{x}^{k}$ for all $k \geq i$ and $G_{x, r}^{k} \unlhd G_{x}^{k}$ for all $r \geq 0$, to show the first statement we have only to prove that for any $k<i$ and each $g \in G_{x, s_{i-1}}^{i}$ and $h \in G_{x, s_{k-1}+}^{k}$, that $g^{-1} h g \in H_{+}$. Recall that a property of the Moy-Prasad filtration is that $\left[G_{x, r}^{k}, G_{x, s}^{k}\right] \subset G_{x, r+s}^{k}$ for all $r, s \in \mathbb{R} \cup \mathbb{R}+, r, s \geq 0$; see for example [HM08, §2]. Therefore since $G^{k} \subset G^{i}$ and $s_{k-1}+>0,\left[h^{-1}, g^{-1}\right] \subset G_{x, s_{i-1}+}^{i}$, whence $g^{-1} h g \in h G_{x, s_{i-1}+}^{i} \subset H_{+}$. Since $J^{i} \subset J \subset G_{x}$ and for any $t>0$ the group $G_{x, t}$ is normal in $G_{x}$, the lemma follows.

For each $i$, let $Z^{i}$ denote the centre of $G^{i}$. For $t>0$, let $i$ be the largest index such that $s_{i-1}<t$, so that $H_{t}=G_{x, t} G_{x, s_{i}+}^{i+1} \cdots G_{x, s_{d-1}+}^{d}$. Let

$$
\Theta_{t}=\mathscr{B}(G)^{H_{t+}} \backslash \mathscr{B}(G)^{Z_{t}^{i}}
$$

be the set of points in $\mathscr{B}(G)$ which are fixed by $H_{t+}$ but not by the subgroup $Z_{t}^{i}$ of $H_{t}$. This is empty if $t>s_{d-1}$, since then $i=d$ and $Z_{t}^{d}$ fixes $\mathscr{B}(G)$ pointwise. Let

$$
\Theta=\bigcup_{0 \leq t \leq s_{d-1}} \Theta_{t}
$$

Theorem 9.2. Suppose that $g \in G$ is such that $\Theta \cap\left[x, g^{-1} y\right] \neq \emptyset$. Then no irreducible subrepresentation of the Mackey component ${ }^{g} \tau(y, g)$ may be a $[G, \pi]_{G^{-}}$ type.

More specifically, we deduce that for every irreducible component $\tau$ of $\tau(y, g)$, there exists an inertial equivalence class $\mathfrak{t} \neq[G, \pi]_{G}$ and an irreducible representation $\pi^{\prime}$ of inertial support $\mathfrak{t}$ such that $\operatorname{Hom}_{G_{g^{-1}}}\left(\tau, \pi^{\prime}\right) \neq 0$.

Proof. Given $g \in G$ such that $\Theta \cap\left[x, g^{-1} y\right] \neq \emptyset$, there exists $0<t \leq s_{d-1}$ such that we may choose a point $u \in \Theta_{t} \cap\left[x, g^{-1} y\right]$. Let $0 \leq i<d$ be maximal with respect to the property that $s_{i-1}<t$.

Since $u \in\left[x, g^{-1} y\right]$, we have $J \cap G_{g^{-1} y} \subset J \cap G_{u}$ and $H_{t} \cap G_{g^{-1} y} \subset H_{t} \cap G_{u}$. Since $u \in \mathscr{B}(G)^{H_{t+}} \backslash \mathscr{B}(G)^{Z_{t}^{i}}$, we have $H_{t+} \subset G_{u}$ but $Z_{t}^{i} H_{t+} \not \subset G_{u}$. Therefore we may 
choose an element $z \in Z_{t}^{i}$ such that $z H_{t+} \not \subset G_{u}$. Since $t \in\left(s_{i-1}, s_{i}\right]$, the quotient $H_{t} / H_{t+}$ is isomorphic to the abelian group $G_{x, t: t+}^{i}$.

Let $\mu$ be a character of $H_{t} / H_{t+}$ which is trivial on $\left(H_{t} \cap G_{u}\right) H_{t+}$ but non-trivial on $z H_{t+}$. Inflate $\mu$ to a character of $H_{t}$. Write $\theta_{t}=\left.\theta_{\Sigma}\right|_{H_{t}}$, and set $\theta^{\prime}=\theta_{t} \mu$. Then $\theta_{t}$ and $\theta^{\prime}$ are characters of $H_{t}$ with the following properties:

(i) $\theta_{t}=\theta^{\prime}$ on $H_{t} \cap G_{u} \supset H_{t} \cap G_{g^{-1} y}$;

(ii) $\theta_{t}=\theta^{\prime}$ on $H_{t+}$; and

(iii) $\theta_{t}(z) \neq \theta^{\prime}(z)$.

Suppose that $\tau$ is an irreducible subrepresentation of the twisted Mackey component $\tau(y, g)$, so that by Frobenius reciprocity we have

$$
0 \neq \operatorname{Hom}_{G_{g^{-1}}}\left(\tau, \operatorname{Ind}_{J \cap G_{g^{-1} y}}^{G_{g^{-1} y}} \lambda\right)=\operatorname{Hom}_{G_{g^{-1}} \cap J}(\tau, \lambda) .
$$

It follows that $\tau$ and $\lambda$ intertwine on the smaller subgroup $G_{g^{-1} y} \cap H_{t}$, where $\left.\lambda\right|_{H_{t}}$ is $\theta_{t}$-isotypic. Applying Frobenius reciprocity again, we have

$$
0 \neq \operatorname{Hom}_{G_{g^{-1}}} \cap H_{t}\left(\tau, \theta_{t}\right)=\operatorname{Hom}_{H_{t}}\left(\operatorname{Ind}_{G_{g^{-1}} \cap H_{t}}^{H_{t}} \tau, \theta_{t}\right) .
$$

By (i), we can replace $\theta_{t}$ by $\theta^{\prime}$ on $G_{g^{-1} y} \cap H_{t}$, so that (9.1) is equivalent to the statement

$$
0 \neq \operatorname{Hom}_{G_{g^{-1}}} \cap H_{t}\left(\tau, \theta^{\prime}\right)=\operatorname{Hom}_{H_{t}}\left(\operatorname{Ind}_{G_{g^{-1}}}^{H_{t}} \cap H_{t} \tau, \theta^{\prime}\right) .
$$

Now, and for the remainder of the proof, suppose to the contrary that $\left(G_{g^{-1} y}, \tau\right)$ is $[G, \pi]_{G}$-typical. Since $[G, \pi]_{G}$ is a supercuspidal inertial equivalence class, this is equivalent to assuming that $\left(G_{g^{-1} y}, \tau\right)$ is a $[G, \pi]_{G^{-}}$type. In particular, this allows us to apply BK98, Prop. 5.2] to deduce that any irreducible subquotient of $\pi_{\tau}:=\mathrm{c}-\operatorname{Ind}_{G_{g^{-1} y}}^{G} \tau$ is isomorphic to the twist of $\pi$ by some unramified character of $G$.

We recognize the representation $\operatorname{Ind}_{G_{g}-1}^{H_{t}} \cap H_{t} \tau$ appearing in (9.1) and (9.2) as a Mackey component of $\operatorname{Res}_{H_{t}}^{G} \pi_{\tau}$, which is by the preceding a direct sum of copies of $\operatorname{Res}_{H_{t}}^{G} \pi$. It therefore follows that the characters $\theta_{t}$ and $\theta^{\prime}$ both occur in $\operatorname{Res}_{H_{t}}^{G} \pi$. Given that

$$
\operatorname{Res}_{H_{t}}^{G} \pi=\bigoplus_{a \in H_{t} \backslash G / J} \operatorname{Ind}_{H_{t} \cap^{a} J}^{H_{t}} \lambda
$$

we may choose $a \in G$ such that the corresponding component contains $\theta^{\prime}$. We then have

$$
0 \neq \operatorname{Hom}_{H_{t}}\left(\theta^{\prime}, \operatorname{Ind}_{H_{t} \cap^{a}{ }_{J}}^{H_{t}} \lambda\right)=\operatorname{Hom}_{H_{t} \cap^{a} J}\left(\theta^{\prime},{ }^{a} \lambda\right) .
$$

Therefore, on the smaller subgroup $H_{t} \cap{ }^{a} H_{t}$, where ${ }^{a} \lambda$ is ${ }^{a} \theta_{t}$-isotypic, we have

$$
\operatorname{Hom}_{H_{t} \cap a} H_{t}\left(\theta^{\prime},{ }^{a} \theta_{t}\right) \neq 0 .
$$

In other words, $a \in G$ intertwines the two characters $\theta_{t}$ and $\theta^{\prime}$ on $H_{t}$.

We claim that to complete the proof, we need only to show that we may choose $a \in G^{i}$. Indeed, since $Z^{i}$ is central in $G^{i}$, we would therefore have that $Z_{t}^{i} \subseteq H_{t} \cap{ }^{a} H_{t}$ and that the characters $\theta^{\prime}$ and $\theta_{t}$ agree on $Z_{t}^{i}$, contradicting (iii) above. Whence it follows that $\tau$ cannot be a $[G, \pi]_{G^{-}}$type.

To show that indeed such an $a \in G^{i}$ exists, we carry out a variant of an inductive argument appearing in [Hak18] and Yu01] that is central to the proof that $(J, \lambda)$ is a $[G, \pi]_{G}$-type. 
Suppose that $i \leq j<d$ and $a \in G^{j+1}$ intertwines $\theta_{t}$ and $\theta^{\prime}$ on $H_{t}$. That is, $a$ satisfies

$$
\operatorname{Hom}_{H_{t} \cap{ }^{a} H_{t}}\left(\theta^{\prime},{ }^{a} \theta_{t}\right) \neq 0 .
$$

Since by condition (ii) $\theta_{t}=\theta^{\prime}$ on $H_{t+}$, when we restrict to $H_{t+} \cap{ }^{a} H_{t+}$ we can replace $\theta^{\prime}$ by $\theta_{t}$ to conclude that

$$
\operatorname{Hom}_{H_{t+} \cap^{a} H_{t+}}\left(\theta_{t},{ }^{a} \theta_{t}\right) \neq 0 .
$$

This intertwining implies that

$$
\left[a^{-1}, H_{t+}\right] \cap H_{t+} \subset \operatorname{ker} \theta_{t} \subset \operatorname{ker} \theta .
$$

Recall that $H_{+}$contains the subgroups $\partial_{+}^{i}$ of (5.2) for any $0<i \leq d$. Since $t \leq s_{i} \leq s_{j}$, we have that $\mathcal{J}_{+}^{j+1} \subset H_{s_{j}+} \subset H_{t+}$, whence

$$
\left[a^{-1}, \partial_{+}^{j+1}\right] \cap \partial_{+}^{j+1} \subset \operatorname{ker} \theta .
$$

Since $a \in G^{j+1}$ and ${\partial_{+}^{j+1}} \subset G^{j+1}$, this is a subgroup of $G^{j+1 \text {,der }} \cap \mathcal{J}_{+}^{j+1}$. By Hak18, Lemma 3.9.1(5)], the character $\theta$ coincides with the character $\hat{\phi}^{j}$ on this intersection. Consequently $\hat{\phi}^{j}$ is also trivial on $\left[a^{-1}, \partial_{+}^{j+1}\right] \cap \mathcal{\partial}_{+}^{j+1}$, which implies $a$ intertwines $\hat{\phi}^{j}$, that is,

$$
\operatorname{Hom}_{\mathcal{J}_{+}^{j+1} \cap^{a} \mathcal{J}_{+}^{j+1}}\left(\hat{\phi}^{j},{ }^{a} \hat{\phi}^{j}\right) \neq 0 .
$$

Applying [Yu01, Thm 9.4], we infer that $a \in j^{j+1} G^{j} \jmath^{j+1}$. Thus there exist $j_{1}, j_{2} \in$ $j^{j+1}$ and $b \in G^{j}$ such that $a=j_{1} b j_{2}$. We wish to show that $b$ intertwines $\theta^{\prime}$ and $\theta_{t}$ on $H_{t}$, which is to say that $\theta^{\prime}$ and ${ }^{b} \theta_{t}$ agree on $H_{t} \cap{ }^{b} H_{t}$.

So let $h \in H_{t} \cap{ }^{b} H_{t}$. Noting that $b^{-1} h b \in H_{t}$ and that $\lambda$ is $\theta_{t}$-isotypic on $H_{t}$ we have, as operators on the space of $\lambda$, that

$$
\begin{aligned}
{ }^{b} \theta_{t}(h) & =\theta_{t}\left(b^{-1} h b\right)=\theta_{t}\left(j_{2} a^{-1} j_{1} h j_{1}^{-1} a j_{2}^{-1}\right) \\
& =\lambda\left(j_{2} a^{-1} j_{1} h j_{1}^{-1} a j_{2}^{-1}\right) \\
& =\lambda\left(j_{2}\right){ }^{a} \lambda\left(j_{1} h j_{1}^{-1}\right) \lambda\left(j_{2}^{-1}\right) .
\end{aligned}
$$

Since $j_{1}, j_{2} \in J^{j+1} \subset J^{j+1}$ normalize $H_{t}$ by Lemma 9.1 we have

$$
j_{1} h j_{1}^{-1} \in{ }^{j_{1}}\left(H_{t} \cap{ }^{b} H_{t}\right)=H_{t} \cap{ }^{j_{1} b} H_{t}=H_{t} \cap{ }^{a} H_{t},
$$

whence ${ }^{a} \lambda\left(j_{1} h j_{1}^{-1}\right)$ acts by the scalar ${ }^{a} \theta_{t}\left(j_{1} h j_{1}^{-1}\right)$, which commutes with $\lambda\left(j_{2}\right)$. Since $\theta^{\prime}$ and ${ }^{a} \theta_{t}$ agree on $H_{t} \cap{ }^{a} H_{t}$, we conclude that

$$
{ }^{b} \theta_{t}(h)={ }^{a} \theta_{t}\left(j_{1} h j_{1}^{-1}\right)=\theta^{\prime}\left(j_{1} h j_{1}^{-1}\right) .
$$

Now recall that $\mu=\theta_{t}^{-1} \theta^{\prime}$ is a character of $H_{t}$ which is trivial on $H_{t+}$. When $t \neq s_{i}$, we have $J_{t} / J_{t+}=H_{t} / H_{t+}$, and so $\mu$ inflates to a character of $J_{t}$. If $t=s_{i}$, then $H_{s_{i}} / H_{s_{i}+}=G_{x, s_{i}}^{i} G_{x, s_{i}+}^{i+1} / G_{x, s_{i}+}^{i+1}$, which is a subgroup of the abelian group $J_{s_{i}} / J_{s_{i}+}=G_{x, s_{i}: s_{i}+}^{i+1}$. Thus we can extend $\mu$ to a character of $J_{s_{i}}$ which is trivial on $J_{s_{i}+}$.

Consider now the representation of $J_{t}$ given by $\lambda^{\prime}=\mu \otimes \operatorname{Res}_{J_{t}}^{J} \lambda$. Upon restriction to $H_{t}$, this representation is $\mu \theta_{t}=\theta^{\prime}$-isotypic. Since $t \leq s_{i} \leq s_{j}$, we have that $\mathrm{j}^{j+1} \subset J_{t}$, whence

$$
\theta^{\prime}\left(j_{1} h j_{1}^{-1}\right)=\lambda^{\prime}\left(j_{1}\right) \theta^{\prime}(h) \lambda^{\prime}\left(j_{1}^{-1}\right)=\theta^{\prime}(h) .
$$

Therefore we have found an element $b \in G^{j}$ for which $\theta^{\prime}$ and ${ }^{b} \theta_{t}$ agree on $H_{t} \cap{ }^{b} H_{t}$. 
Hence by induction, we may assume that we are given an $a \in G^{i}$ such that $\theta^{\prime}$ and ${ }^{a} \theta_{t}$ agree on $H_{t} \cap{ }^{a} H_{t}$. As argued above, this leads to a contradiction; therefore no $a \in G$ can intertwine $\theta_{t}$ and $\theta^{\prime}$, which implies in turn that $\tau$ cannot be a $[G, \pi]_{G}$-type.

The set $\Theta$, through which the geodesic $\left[x, g^{-1} y\right]$ should pass in order to apply Theorem 9.2. can be described in terms of fixed point sets of compact open groups acting on $\mathscr{B}(G)$, as follows. However, since the geometry of such fixed point sets is an open problem, our description is incomplete.

For each $i, Z_{0}^{i}$ fixes $\mathscr{B}\left(G^{i}\right)$ as a subset of $\mathscr{B}(G)$, and thus the filtration subgroup $Z_{t}^{i}$ fixes a $G^{i}$-invariant convex neighbourhood of $\mathscr{B}\left(G^{i}\right)$. Since we have an inclusion of the compact open subgroups

$$
H_{t+} \subseteq Z_{t}^{i} H_{t+}
$$

it follows that

$$
\mathscr{B}(G)^{H_{t+}} \supseteq \mathscr{B}(G)^{Z_{t}^{i} H_{t+}} .
$$

Their images in the reduced building are bounded convex neighbourhoods of $[x]$ since the groups are compact open and contained in $G_{x}$. Note that $\Theta_{t}$ is their set-theoretic difference, since

$$
\Theta_{t}=\mathscr{B}(G)^{H_{t+}} \backslash \mathscr{B}(G)^{Z_{t}^{i}}=\mathscr{B}(G)^{H_{t+}} \backslash \mathscr{B}(G)^{Z_{t}^{i} H_{t+}} .
$$

This difference can be empty, such as when $t$ is such that $Z_{t}^{i}=Z_{t+}^{i} \subset H_{t+}$. When nonempty, it never meets $\mathscr{B}\left(G^{i}\right)$. One expects that as $t$ varies over the discretely many values in an interval of length 1 at which $Z_{t}^{0} \neq Z_{t+}^{0}$, the regions $\Theta_{t}$ are nonempty and cover the radial directions from each of the buildings $\mathscr{B}\left(G^{i}\right)$, that is, meet $[x, y]$ for all $y \in \operatorname{proj}_{\mathscr{B}\left(G^{i}\right)}^{-1}(\widetilde{[x]})$ sufficiently far from $x$.

In particular, by this reasoning, when $i=0$ and $s_{0}>1$ one expects that for every $z \in \Delta=\operatorname{proj}_{\mathscr{B}\left(G^{0}\right)}^{-1}(\widetilde{[x]})$, outside of the neighbourhood of simplicial radius 1 that

$$
[x, z] \cap\left(\bigcup_{0<t<s_{0}} \Theta_{t}\right) \neq \emptyset
$$

whence Theorem 9.2 would apply to all Mackey components omitted from Theorem 7.1 save those in a small neighbourhood of $[x]$, reducing the question of unicity to types to a finite set of representations of $G_{y}$.

What is required to make this precise in fully generality are bounds on the rate of growth of these fixed point sets in various radial directions as $t$ increases. While the fixed point set of $G_{x, r}$ can be approximated by neighbourhoods of simplifical radius $r$ about $\widetilde{[x]}$ (see [Nev14, Prop 3.3], for example), unfortunately, there is a scarcity of results about the fixed points of (non-maximal) tori and their filtration subgroups. The strongest results of which we are aware are those of [MS12, §4], which use root-theoretic methods to produce upper bounds on the simplicial radius of $\mathscr{B}(G)^{Z_{t}^{i}}$ when $Z^{i}$ is a maximal torus in $G$. These arguments do not seem readily modifiable to the non-maximal case required here, and consequently this seems to be an interesting open question.

Nevertheless, the results of Theorems 7.1 and 9.2 may concretely be applied to any particular $G$, and supercuspidal representation $\pi$, where the nature of the sets $\Delta$ and $\Theta_{t}$ can be explicitly determined. 


\section{ACKNOWLEDGMENTS}

The authors gratefully acknowledge the support of both the Centre International de Rencontres Mathématiques (Luminy) and the Mathematisches Forschungsinstitut Oberwolfach, who hosted them for two-week Research in Pairs stays in 2018 and 2019, respectively. The warm hospitality of these institutes made this work possible.

\section{REFERENCES}

[Ad198] Jeffrey D. Adler, Refined anisotropic K-types and supercuspidal representations, Pacific J. Math. 185 (1998), no. 1, 1-32, DOI 10.2140/pjm.1998.185.1. MR.1653184

[Ber84] J. N. Bernstein, Le "centre" de Bernstein (French), Representations of reductive groups over a local field, Travaux en Cours, Hermann, Paris, 1984, pp. 1-32. Edited by P. Deligne. MR771671

[BK93] Colin J. Bushnell and Philip C. Kutzko, The admissible dual of GL( $N)$ via compact open subgroups, Annals of Mathematics Studies, vol. 129, Princeton University Press, Princeton, NJ, 1993, DOI 10.1515/9781400882496. MR.1204652

[BK98] Colin J. Bushnell and Philip C. Kutzko, Smooth representations of reductive p-adic groups: structure theory via types, Proc. London Math. Soc. (3) 77 (1998), no. 3, 582634, DOI 10.1112/S0024611598000574. MR 1643417

[Blo05] Corinne Blondel, Quelques propriétés des paires couvrantes (French), Math. Ann. 331 (2005), no. 2, 243-257, DOI 10.1007/s00208-004-0579-1. MR2115455

[BLR90] Siegfried Bosch, Werner Lütkebohmert, and Michel Raynaud, Néron models, Ergebnisse der Mathematik und ihrer Grenzgebiete (3) [Results in Mathematics and Related Areas (3)], vol. 21, Springer-Verlag, Berlin, 1990, DOI 10.1007/978-3-642-51438-8. MR1045822

[Bor98] Mikhail Borovoi, Abelian Galois cohomology of reductive groups, Mem. Amer. Math. Soc. 132 (1998), no. 626, viii+50, DOI 10.1090/memo/0626. MR.1401491

[BT72] F. Bruhat and J. Tits, Groupes réductifs sur un corps local (French), Inst. Hautes Études Sci. Publ. Math. 41 (1972), 5-251. MR327923

[BT84] F. Bruhat and J. Tits, Groupes réductifs sur un corps local. II. Schémas en groupes. Existence d'une donnée radicielle valuée (French), Inst. Hautes Études Sci. Publ. Math. 60 (1984), 197-376. MR 756316

[Cas73] William Casselman, The restriction of a representation of $\mathrm{GL}_{2}(k)$ to $\mathrm{GL}_{2}(\mathfrak{o})$, Math. Ann. 206 (1973), 311-318, DOI 10.1007/BF01355984. MR338274

[Fin19] Jessica Fintzen, On the Moy-Prasad filtration, J. Eur. Math. Soc. (JEMS) 23 (2021), no. 12, 4009-4063, DOI 10.4171/jems/1098. MR4321207

[Fin21] Jessica Fintzen, Types for tame p-adic groups, Ann. of Math. (2) 193 (2021), no. 1, 303-346, DOI 10.4007/annals.2021.193.1.4. MR.4199732

[Hak18] Jeffrey Hakim, Constructing tame supercuspidal representations, Represent. Theory 22 (2018), 45-86, DOI 10.1090/ert/514. MR3817964

[HM08] Jeffrey Hakim and Fiona Murnaghan, Distinguished tame supercuspidal representations, Int. Math. Res. Pap. IMRP 2 (2008), Art. ID rpn005, 166. MR2431732

[Kal19] Tasho Kaletha, Regular supercuspidal representations, J. Amer. Math. Soc. 32 (2019), no. 4, 1071-1170, DOI 10.1090/jams/925. MR4013740

[Kim07] Ju-Lee Kim, Supercuspidal representations: an exhaustion theorem, J. Amer. Math. Soc. 20 (2007), no. 2, 273-320, DOI 10.1090/S0894-0347-06-00544-3. MR2276772

[Kot97] Robert E. Kottwitz, Isocrystals with additional structure. II, Compositio Math. 109 (1997), no. 3, 255-339, DOI 10.1023/A:1000102604688. MR.1485921

[Kut77] P. C. Kutzko, Mackey's theorem for nonunitary representations, Proc. Amer. Math. Soc. 64 (1977), no. 1, 173-175, DOI 10.2307/2041005. MR442145

[KY17] Ju-Lee Kim and Jiu-Kang Yu, Construction of tame types, Representation theory, number theory, and invariant theory, Progr. Math., vol. 323, Birkhäuser/Springer, Cham, 2017, pp. 337-357. MR3753917

[Lat16] Peter Latham, Unicity of types for supercuspidal representations of p-adic $\mathbf{S L}_{2}$, J. Number Theory 162 (2016), 376-390, DOI 10.1016/j.jnt.2015.10.008. MR3448273

[Lat17] Peter Latham, The unicity of types for depth-zero supercuspidal representations, Represent. Theory 21 (2017), 590-610, DOI 10.1090/ert/511. MR.3735454 
[Lat18] Peter Latham, On the unicity of types in special linear groups, Manuscripta Math. 157 (2018), no. 3-4, 445-465. MR3858412

[LN19] Peter Latham and Monica Nevins, On the unicity of types for toral supercuspidal representations, Representations of reductive p-adic groups, Progr. Math., vol. 328, Birkhäuser/Springer, Singapore, 2019, pp. 175-190. MR3889763

[Mor99] Lawrence Morris, Level zero G-types, Compositio Math. 118 (1999), no. 2, 135-157, DOI 10.1023/A:1001019027614. MR1713308

[MP96] Allen Moy and Gopal Prasad, Jacquet functors and unrefined minimal K-types, Comment. Math. Helv. 71 (1996), no. 1, 98-121, DOI 10.1007/BF02566411. MR.1371680

[MS12] Ralf Meyer and Maarten Solleveld, Characters and growth of admissible representations of reductive p-adic groups, J. Inst. Math. Jussieu 11 (2012), no. 2, 289-331, DOI 10.1017/S1474748011000120. MR2905306

[Nad17] Santosh Nadimpalli, Typical representations for level zero Bernstein components of $\mathrm{GL}_{n}(F)$, J. Algebra 469 (2017), 1-29, DOI 10.1016/j.jalgebra.2016.08.033. MR.3563005

[Nad19] Santosh Nadimpalli, On classification of typical representations for $\mathrm{GL}_{3}(F)$, Forum Math. 31 (2019), no. 4, 917-941, DOI 10.1515/forum-2019-0031. MR3975668

[Nev13] Monica Nevins, Branching rules for supercuspidal representations of $\mathrm{SL}_{2}(k)$, for $k$ a p-adic field, J. Algebra 377 (2013), 204-231, DOI 10.1016/j.jalgebra.2012.12.003. MR3008903

[Nev14] Monica Nevins, On branching rules of depth-zero representations, J. Algebra 408 (2014), 1-27, DOI 10.1016/j.jalgebra.2014.03.016. MR3197168

[Pas05] Vytautas Paskunas, Unicity of types for supercuspidal representations of $\mathrm{GL}_{N}$, Proc. London Math. Soc. (3) 91 (2005), no. 3, 623-654, DOI 10.1112/S0024611505015340. MR2180458

[PR08] G. Pappas and M. Rapoport, Twisted loop groups and their affine flag varieties, Adv. Math. 219 (2008), no. 1, 118-198, DOI 10.1016/j.aim.2008.04.006. With an appendix by T. Haines and Rapoport. MR2435422

[SS08] V. Sécherre and S. Stevens, Représentations lisses de $\mathrm{GL}_{m}(D)$. IV. Représentations supercuspidales (French, with English and French summaries), J. Inst. Math. Jussieu 7 (2008), no. 3, 527-574, DOI 10.1017/S1474748008000078. MR2427423

[Ste05] Shaun Stevens, Semisimple characters for p-adic classical groups, Duke Math. J. 127 (2005), no. 1, 123-173, DOI 10.1215/S0012-7094-04-12714-9. MR2126498

[Ste08] Shaun Stevens, The supercuspidal representations of p-adic classical groups, Invent. Math. 172 (2008), no. 2, 289-352, DOI 10.1007/s00222-007-0099-1. MR2390287

[Yu01] Jiu-Kang Yu, Construction of tame supercuspidal representations, J. Amer. Math. Soc. 14 (2001), no. 3, 579-622, DOI 10.1090/S0894-0347-01-00363-0. MR1824988

[Yu15] Jiu-Kang Yu, Smooth models associated to concave functions in Bruhat-Tits theory (English, with English and French summaries), Autour des schémas en groupes. Vol. III, Panor. Synthèses, vol. 47, Soc. Math. France, Paris, 2015, pp. 227-258. MR 3525846

Department of Mathematics and Statistics, University of Ottawa, Ottawa, Canada Email address: platham@uottawa.ca

Department of Mathematics and Statistics, University of Ottawa, Ottawa, Canada

Email address: mnevins@uottawa.ca 